\title{
ExoClock project: an open platform for monitoring the ephemerides of Ariel targets with contributions from the public
}

\author{
Anastasia Kokori ${ }^{1,2} \cdot$ Angelos Tsiaras $^{1,2} \cdot$ Billy Edwards $^{1} \cdot$ Marco Rocchetto $^{1,3}$. \\ Giovanna Tinetti ${ }^{1}$ - Anaël Wünsche ${ }^{4}$. Nikolaos Paschalis ${ }^{5}$. \\ Vikrant Kumar Agnihotri ${ }^{6}$. Matthieu Bachschmidt ${ }^{7}$. Marc Bretton ${ }^{4}$. \\ Hamish Caines $^{1}$. Mauro Caló ${ }^{32}$. Roland Casali ${ }^{8}$. Martin Crow ${ }^{9,10}$. \\ Simon Dawes ${ }^{9,10}$. Marc Deldem ${ }^{11}$. Dimitrios Deligeorgopoulos ${ }^{12}$. \\ Roger Dymock ${ }^{9}$. Phil Evans ${ }^{13}$. Carmelo Falco ${ }^{14}$. Stephane Ferratfiat ${ }^{4}$. \\ Martin Fowler $^{9}$. Stephen Futcher ${ }^{15}$. Pere Guerra ${ }^{16}$. Francois Hurter ${ }^{17}$. \\ Adrian Jones $^{9}$. Wonseok Kang ${ }^{18} \cdot$ Taewoo Kim $^{18}$. Richard Lee ${ }^{7}$. \\ Claudio Lopresti ${ }^{19}$. Antonio Marino ${ }^{20}$. Matthias Mallonn ${ }^{21}$. Fabio Mortari ${ }^{22}$. \\ Mario Morvan ${ }^{1}$ - Lorenzo V. Mugnai ${ }^{33}$. Alessandro Nastasi ${ }^{14}$. \\ Valère Perroud ${ }^{23}$. Cédric Pereira ${ }^{34}$. Mark Phillips ${ }^{9,24}$. Pavel Pintr ${ }^{25}$. \\ Manfred Raetz $^{26}$. Francois Regembal ${ }^{27}$. John Savage ${ }^{9} \cdot$ Danilo Sedita $^{28}$. \\ Nick Sioulas ${ }^{29}$. lakovos Strikis ${ }^{30}$. Geoffrey Thurston ${ }^{9}$. Andrea Tomacelli ${ }^{20}$. \\ Alberto Tomatis ${ }^{31}$
}

Received: 10 July 2020 / Accepted: 16 December 2020/Published online: 11 August 2021

(C) The Author(s) 2021

\begin{abstract}
The Ariel mission will observe spectroscopically around 1000 exoplanets to further characterise their atmospheres. For the mission to be as efficient as possible, a good knowledge of the planets' ephemerides is needed before its launch in 2028. While ephemerides for some planets are being refined on a per-case basis, an organised effort to collectively verify or update them when necessary does not exist. In this study, we introduce the ExoClock project, an open, integrated and interactive platform with the purpose of producing a confirmed list of ephemerides for the planets that will be observed by Ariel. The project has been developed in a manner to make the best use of all available resources: observations reported in the literature, observations from space instruments and, mainly, observations from ground-based telescopes, including both professional and amateur observatories. To facilitate inexperienced observers and at the same time achieve homogeneity in the results, we
\end{abstract}

Anastasia Kokori

anastasia.kokori@gmail.com

Extended author information available on the last page of the article. 
created data collection and validation protocols, educational material and easy to use interfaces, open to everyone. ExoClock was launched in September 2019 and now counts over 140 participants from more than 15 countries around the world. In this release, we report the results of observations obtained until the 15h of April 2020 for 120 Ariel candidate targets. In total, 632 observations were used to either verify or update the ephemerides of 84 planets. Additionally, we developed the Exoplanet Characterisation Catalogue (ECC), a catalogue built in a consistent way to assist the ephemeris refinement process. So far, the collaborative open framework of the ExoClock project has proven to be highly efficient in coordinating scientific efforts involving diverse audiences. Therefore, we believe that it is a paradigm that can be applied in the future for other research purposes, too.

Keywords Exoplanets · Ephemerides · Open science · Data analysis · Photometry · Citizen science

\section{Introduction}

Just after discovery, the time of the next transit for a planet is well known. However, the accuracy of predicted future transits degrades over time due to the increased number of epochs since the last observation and the stacking of the period error (e.g. [1-3]). In extreme cases this can mean the transit time is practically lost, with errors of several hours (e.g. [4]). In addition to this issue, extrapolating transit times from only a few data points over a limited baseline can easily introduce bias (e.g. [1,5]). Finally, we could expect transit times to shift due to dynamical phenomena such as tidal orbital decay, apsidal precession or from gravitational interactions with other bodies in the system (e.g. [6-8]).

These issues can only be understood, and mitigated for, by regular observations over a long baseline and continuous ephemeris refinement. In this effort, small telescopes have been proven to be as efficient as larger ones (e.g. [1, 3, 9-11]). Especially for targets that are being discovered at the moment by TESS, estimations suggest that we will need efficient follow-up observations to verify their ephemerides and narrow down the large uncertainties in the predicted transit times [2, 3].

The Ariel mission [12] will spectroscopically observe 1000 targets to study their atmospheres. To achieve this goal, thousands of transits will be observed within the lifetime of the mission. This large number underlines the necessity for precise predictions of the transit times, in order to maximise the overall efficiency of the mission.

When planning observations for a single planet or for a small number of planets, ephemerides updates can be done on a per-case basis. However, in the new era of characterising large numbers of planets, such an effort needs to be organised in a much more efficient way through an open and collaborative platform, in order to make the best use of all the currently available resources. In several fields of astronomy there are public and collaborative databases which contribute significantly to 
community building and sharing. Examples of such efforts in astronomical fields are: VESPA $^{1}$ [13], the Minor Planet Center ${ }^{2}$ and Simbad. ${ }^{3}$

In this work, we present the ExoClock project (https://www.exoclock.space), an open, integrated platform with the scope of verifying the ephemerides of the planets that will be observed by Ariel, prior to the launch of the mission. ExoClock has been designed in a way to make the best use of all the available resources, including literature observations regardless of their purpose, and observations from any available ground- or space-based facilities, smaller or larger in size. In addition, ExoClock will provide a continuously updated and homogenous ephemerides service, which can be valuable for general exoplanet research, even beyond the Ariel mission.

\section{The ExoClock project}

Since Ariel will observe thousands of transits, good knowledge of the transit ephemerides is important in order to maximise the efficiency of the observing schedule and of the mission in general. The idea of the ExoClock project was conceptualised to achieve this scope, by delivering a verified list of ephemerides for the planets in the Ariel candidate target list [14], before the launch of Ariel. At the moment, this list includes about two thousand planets, from which 370 are already known and the rest are simulated future discoveries. From these planets, around 1000 will be chosen and observed by Ariel.

As the Ariel target candidate list includes thousands of planets, to achieve the goal of verifying their ephemerides we need to make the best use of all currently available resources and collect a large number of observations throughout many years. The ExoClock project has been designed to implement the above approach by:

1. integrating dedicated follow-up observations conducted by observers that have joined the project (forming the ExoClock network), literature observations, and observations conducted for other purposes, both from ground and space;

2. being open to contributions from a variety of audiences - professional and amateur astronomers, students, and industry partners;

3. interacting with the participants through a variety of user-friendly interfaces to efficiently coordinate their efforts and ensure the high quality of the data products.

The diversity of the project requires strategies to ensure homogeneity and reliability in the results. To achieve this vision, we have planned and applied several strategies which will be discussed in detail later. Since the project is open to contributions from inexperienced observers and at the same time contributions from various sources, it is very important to provide user-friendly interfaces and educational material. Throughout the course of the ExoClock project, we accomplish not only the

\footnotetext{
${ }^{1}$ http://www.europlanet-vespa.eu

${ }^{2}$ https://www.minorplanetcenter.net/iau/mpc.html

${ }^{3}$ http://simbad.u-strasbg.fr/simbad
} 
most efficient use of resources but also a more vital connection between the several partners that collaborate. The open framework is an essential element in order to cocreate knowledge effectively and share it with the rest of the community. ExoClock observations will be shared with the community every time a sufficient number of observations is collected.

\subsection{The citizen science aspects of ExoClock}

Since ExoClock is open to contributions from everyone, inexperienced participants (e.g. amateur astronomers, students, citizen scientists, members of the public) constitute naturally an important part of the project. Currently, there are 140 participants in ExoClock, of which $80 \%$ are amateur astronomers and the rest $20 \%$ includes both early career and senior professional astronomers, but also a few school students and teachers. Therefore, the project has also a citizen science character. There are numerous citizen science projects designed to support scientific research and, indeed, citizen science is a mainstream trend. Citizen Science projects are efficient from various aspects [15], such as:

- large-scale data collection;

- elaborating scientific knowledge;

- increasing the participation of a wider community;

- strengthening the relationship between science and society;

However, several issues need to be considered as many of these projects have been questioned for their credibility and the accuracy in the delivered results. Hence, it is very important to plan and implement citizen science aspects carefully, considering a number of different parameters to ensure scientific robustness. In the ExoClock project we use different activities that are implemented at different stages during the project (data collection, data analysis and data validation). Following recommendations from successful citizen science projects [16-18], we include strategies such as:

- educational material;

- training sections;

- interaction between the participants and the scientific team;

- data collection protocols;

- analysis tools;

- validation to a reference database;

- cross validation;

- interactive project development;

\subsection{The Exoplanet characterisation catalogue (ECC)}

As the final scientific product of this project is a verified catalogue of ephemerides, it was vital to construct an initial catalogue with the scope of continually updating it until Ariel launches. This catalogue was essential for the homogeneity in the final results but also for achieving the most efficient way of planning and analysing the observations collected by the participants. 
The Exoplanet Characterisation Catalogue (ECC) includes the planets of the Ariel candidate target list that have already been discovered [14]. At this moment, it includes 370 planets but it will be continuously updated based on upcoming discoveries. The catalogue contains stellar parameters (RA, DEC, magnitude at different filters, Temperature, Gravity and Metallicity) and also the transit parameters (ephemeris, period, Rp/ Rs, depth, inclination, transit duration and eccentricity). The ECC can be found as part of the data provided in this work (see Section 5).

To ensure consistency in the catalogued values, we constructed the ECC following the rules below:

1. The target parameters (position, and magnitudes) were extracted from:

- SIMBAD for RA, DEC, and the magnitudes V, R, I

- GAIA for the GAIA magnitudes G, G-BP, G-RP

- 2MASS for the 2MASS magnitudes J, H, K

Where any of the V, R, I magnitudes were missing, we used the GAIA magnitudes to calculate them. ${ }^{4}$ This is indicated in the catalogue where applicable.

2. The stellar parameters used to calculate the limb darkening - effective temperature, surface gravity, metallicity -were extracted from a single source for each planet (reference in the catalogue);

3. The transit parameters - planet radius, semi-major axis, eccentricity, inclination, argument of periastron - were extracted from a single source for each planet (reference in the catalogue);

4. The ephemeris parameters - zero-epoch mid-time, period - were extracted from a single source for each planet, the most recent one, but with the scope of updating them in the course of the project (reference in the catalogue).

\subsection{Ephemeris verification criterion}

Observing before and after the transit is important to correct for instrumental systematics and Ariel will observe $75 \%$ of the transit duration before and after each transit. Hence, we have defined that for an ephemeris to be considered as verified, we need to be sure that at least $50 \%$ of the transit duration will be observed both before and after the transit. This is translated to a $3 \sigma$ uncertainty in the transit time prediction for the end of 2028 - lower than $25 \%$ of the transit duration, or to an $1 \sigma$ uncertainty lower than $1 / 12$ of the transit duration (target uncertainty).

\section{Best use of resources}

In order to achieve the goals of the project it is fundamental to make the best use of all available resources. These include the observations available in the literature, and ongoing observations from space- and ground-based facilities, the latter including both professional and amateur observatories.

\footnotetext{
${ }^{4}$ GAIA documentation
} 


\subsection{The ExoClock network of telescopes}

In the ExoClock project we have developed an interface, where interested observers - either professional or amateur astronomers - can join the ExoClock network by registering their telescopes. So far, 140 observers have joined the ExoClock project, registering 180 telescopes to the ExoClock network. These telescopes are located in 15 countries around the world, and are of sizes between 6 and 40 inches.

Given the diversity in both the location and the sizes of the telescopes, we created a prioritisation system and a personalised scheduler for every registered telescope.

\subsubsection{Prioritisation system}

We assigned an observing priority (high, medium, or low) to every planet in the ECC based on the $1 \sigma$ uncertainty in the transit time prediction (1):

$$
\sigma_{T}=\sqrt{\sigma_{T_{0}}^{2}+\left(N \sigma_{P}\right)^{2}}
$$

where $T$ is the mid-time prediction, $T_{0}$ is the ephemeris mid-time, $\mathrm{N}$ is the number of epochs since the $T_{0}$, and $P$ is the orbital period of the planet.

We initially assigned an observing priority to every planet, as follows:

- For planets with $1 \sigma$ uncertainty in the transit time prediction for the end of 2020 higher than $1 / 12$ of the transit duration, the priority was set to high. Otherwise, if the ephemeris was older than four years, again the priority was set to high.

- For the remaining planets, if the $1 \sigma$ uncertainty in the transit time prediction for the end of 2028 was higher than $1 / 12$ of the transit duration, the priority was set to medium. Otherwise, if the ephemeris was between two and four years old, again the priority was set to medium.

- For all the remaining planets, the priority was set to low.

We also included a category of planets marked as "alert", for which new observations suggest a time-shift higher than ten minutes. In this way we encourage observers to follow these specific planets until a clear picture about their ephemerides is formed.

During the course of the project the priorities are being dynamically updated based on the collected observations. For example, if a high priority planet is observed enough times during an observing season to improve or verify the ephemeris, its priority will be reduced to low. However, if the planet is not observed again in the following observing season, its priority will be increased again to medium.

\subsubsection{Personalised schedule}

To allocate more efficiently the targets to different observers, we have created a personalised schedule, which indicates to every observer the transits that are accessible to them, depending on their equipment and their location. 
The expected signal-to-noise ratio is calculated based on (2) and if this is greater than 15 , the planet is marked as observable.

$$
S N R=a D \sqrt{10^{\frac{12-R_{m a g}}{2.5}}} \frac{T_{D p}}{\sqrt{\frac{1}{T_{D r}}+\frac{1}{120}}}
$$

where $a$ is a constant, $D$ is the telescope aperture in inches, $R_{\text {mag }}$ is the R magnitude of the star, $T_{D p}$ is the transit depth in mmag, and $T_{D r}$ is the transit duration in minutes.

The constant $a$ was initially calculated based on observations we acquired from the Holomon Astronomical station of the Aristotle University of Thessaloniki in Greece. They represent a system with an 11 inch aperture, an ATIK11000 camera and a Red (Cousins) filter, which is widely considered as a typical equipment capable of conducting transit observations. For this system and assuming an observation that starts one hour before the transit, ends one hour after the transit, has an exposure time of one minute, and has overheads of 30 seconds, $a$ is equal to 0.0125 .

As observers submit their observations, we track the performance of their systems and further adjust the $a$ coefficient per system. In this way the schedule takes into account the capabilities of the different instruments and the long-term effects of weather patterns, in order to distribute the planets accordingly. In terms of timing, only the observations that happen when the star is at least 20 degrees above the horizon appear in the scheduler. Finally, we make sure that the Sun stays below -10 degrees throughout the observation.

Based on the above calculation, and placing an upper limit for the transit duration to 6 hours, we computed the number of planets in the Ariel candidate target list that are accessible through the ExoClock network. For telescopes of 10, 15, 25, 35, and 40 inches the percentages are $14 \%, 22 \%, 36 \%, 47 \%, 52 \%$, respectively. If we however relax the requirement of $\mathrm{SNR}=15$ to $\mathrm{SNR}=10$, these numbers increase to $22 \%, 33 \%$, $49 \%, 62 \%, 67 \%$, respectively.

\subsection{Space-based facilities}

Space-based follow up will be needed for targets that are not accessible from the ground (of low $\mathrm{S} / \mathrm{N}$ or very long duration). ExoClock will integrate past and future results from the main space telescopes that have observed or will observe planets in the ECC.

\subsubsection{TESS}

During its primary mission, TESS is surveying almost the entire sky. While the prime objective of the mission is to discover new planets, TESS will also observe stars which are known to host planets. As of November 2019, TESS has observed the host stars of 150 planets within the Ariel target list. However, data of sufficient quality has not necessarily been obtained for each of these. Thus, while TESS will undoubtedly be a great resource for the refinement of transit times (e.g. [10, 19, 20]), the extent of this contribution needs further exploration. 


\subsubsection{Hubble and Spitzer}

Spitzer has been used to follow-up several exoplanets with ephemeris uncertainties (e.g. [5, 21, 22]) and the data will be valuable for future ephemeris refinements. However, the spacecraft has ceased operations in early 2020 and no further observations are available. The Hubble Space Telescope (HST) has been delivering spectroscopic observations of exoplanets since 2001. Although most observations are interrupted due to Earth obscuration, the precision can still be sufficient for measuring accurate transit times (e.g. [23, 24]. HST may well be used to observe new TESS detections and could therefore provide ephemeris refinement. However, HST cannot be used in cases where the uncertainty on the transit time is too large. Also, HST is a general observatory and can only observe a limited number of targets. Therefore, neither of these missions can be expected to have a major impact on any ephemeris refinement project.

\subsubsection{CHEOPS}

CHEOPS launched in December 2019 and is anticipated to follow-up a large number of TESS discoveries, refining both planetary and orbital parameters via high precision photometry [25]. The mission has reserved around $20 \%$ of the telescope time for guest observers which equates to 946 orbits, or 1578 hours, in the first year. To allow potential users to explore the capabilities of CHEOPS, a simulator has been developed which has a web interface. ${ }^{5}$

By inputting the star type and magnitude, along with the observation duration, a noise prediction is returned. The web-based simulator has been utilised to understand the performance for ephemeris refinement of planets within the Ariel target list. CHEOPS cannot observe the ecliptic poles, which are the continuous viewing zones for JWST and Ariel, and planets outside the field of regard have been removed. From this exercise, we conclude that additional 300 planets $(15 \%)$ that are accessible to CHEOPS with an SNR above 5 in one observation. Further investigation is needed to ascertain the exact number of targets for which CHEOPS could reduce the transit uncertainties but it would seem likely that the final figure will be of the order of several hundred.

\subsection{Other ground-based networks}

ExoClock as an integrated platform will incorporate all available observations produced by other ground-based networks, too. We are already collaborating with other networks and current projects to both make the best use of all resources and decrease the potential for overlapping efforts. Since homogeneity is key for the ExoCloc project, we are currently examining the data sharing protocols and quality control protocols. Data from such databases will be included in the ExoCloc database in

\footnotetext{
${ }^{5}$ https://cheops.unige.ch/pht2/exposure-time-calculator
} 
future releases after defining the most effective strategy to collect them ensuring validity and accuracy.

Although such networks carry out transit exoplanet observations, they are focused on different aspects, as far as exoplanet ephemerides are concerned. For example, the Exoplanet Transit Database (ETD, [26]) run by the Czech Astronomical Society since 2009, provides more than 10,000 transit light curves for more than 350 exoplanets systems, with the scope of searching for transit time variations. Additionally, Exoplanet Watch [3] is another coordinated effort to collect follow-up observations of exoplanet transits with small telescopes, organised in the USA and focused on targets that will be then observed by the James Webb Space Telescope.

Finally, the ExoClock project is in close collaboration with the Telescope Live ${ }^{6}$ network. Telescope Live is a web application offering end-users the possibility to purchase images obtained on-demand from a network of robotic telescopes. At the time of writing the network consists of eight telescopes distributed across three observatories: a 1m ASA RC-1000AZ, a 0.6m Planewave CDK24 and two 0.5m ASA 500N located at El Sauce Observatory in Chile; a $0.7 \mathrm{~m}$ ProRC 700 and two $0.1 \mathrm{~m}$ Takahashi FSQ-106ED located at IC Astronomy in Spain; a 0.1m Takahashi FSQ-106ED located at Heaven's Mirror Observatory in Australia.

\subsection{Catalogue of literature observations}

Since there is currently no organised and easily accessible database of past observations, conducted by other observatories (ground- or space-based) and recorded in the literature, it is difficult for the scientific community to distinguish which planets need their ephemerides to be updated.

To facilitate easier access to the current literature we have developed an application within the website, in which participants and members of the scientific community can add results from other published works. The application includes all the appropriate conversions between the different time systems used across the different papers to the $\mathrm{BJD}_{\mathrm{TDB}}$ system.

These contributions are validated and they are taken into account in the ephemerides refinement. In this work we include the literature data only for the planets for which an ephemeris update is required. We will continue developing this platform and collect more data from past and new publications, to ensure the best use of all transit observations, even beyond the ExoClock platform.

\section{Scientific robustness and homogeneity of the results}

Ensuring homogeneity and maintaining high quality in the results produced is crucial when multiple observers are participating. To achieve this requirement, it is mandatory to base the analysis on a single catalogue, and this was one of the main reasons

\footnotetext{
${ }^{6}$ https://telescope.live
} 
for constructing the ECC described above. In addition, we have established a protocol for conducting and analysing observations, and specific educational material to support the observers.

\subsection{Observing strategy}

Based on experience from previous observations and collaboration with other experts on exoplanets research, we created an observing strategy to be followed as a suggestion by all our participants (data collection protocol). In this strategy we pay extra attention to ensure the observability of the transits, to maximise the total $\mathrm{S} / \mathrm{N}$ ratio of the light curves and to achieve the highest possible precision in transit timing. More specifically, the observers are recommended to:

- follow the personalised schedule to only follow-up planets that can be observed with their equipment and from their location. (see Section 3.1.2 for more details);

- use an R or I Cousins filter, preferably. Alternatively, a luminance or clear filter is suggested. In general, the precision in the measurement of the mid-time of a transit increases with wavelength. The reasons for this improvement are the weaker stellar activity, the weaker limb darkening effect and the lower background noise;

- set the camera temperature to the lowest available value. This step is necessary to decrease the noise from dark current as much as possible;

- set the binning to one to allow longer exposure times, decreasing in this way the total overheads and increasing the total $\mathrm{S} / \mathrm{N}$;

- keep the number of counts below the point where the camera becomes non-linear. This procedure is suggested to avoid biases in the transit depth caused by the non-linear response of the camera;

- check for at least one good comparison star in the field and adjust it if it is needed;

- observe for an extra hour before the start of the transit and an extra hour after the end of the transit. This strategy is necessary to model efficiently any instrumental systematics (see Section 4.3) for details on the systematics modelling). In addition, it helps capturing unexpected drifts between the predicted and the observed transit mid-times;

- validate the clock of their computer against the network time. A precision of one to two seconds can be easily achieved in this way, and it is enough for the purposes of this project.

\subsection{Data reduction and photometry}

The data collection protocol described above is supported by a dedicated piece of software for data reduction and photometry. We specifically developed this software (the HOlomon Photometric Software ${ }^{7}$ ) to certify both the high quality and the homogeneity of the results but also to ensure accessibility for users of different experience levels, starting from users who had never observed transits before.

${ }^{7}$ https://github.com/ExoWorldsSpies/hops 
The software is developed in Python, allowing community development and easy adaptation to new features. It is also continuously developed in collaboration with amateurs who provide feedback on the user experience and the performance of the software under different circumstances. HOPS supports the users by being user friendly; interactive, with user interfaces; open source; free; geospacial; and compatible with all operational systems.

As far as the data analysis is concerned, HOPS allows the user to reduce and align the data; to select the best comparison stars; to extract the target's light curve; and to perform an MCMC fitting to allow for a first validation of the results. Providing more technical details is beyond the scope of this work and will be discussed in a separate, dedicated, publication.

\subsection{Light curve fitting}

While the data reduction and photometry can be performed using the observer's preferred tools, it is of great importance to perform the light curve modelling on the ExoClock web-server. We use the open source Python package PyLightcurve ${ }^{8}$ [27] to facilitate this process.

At first, the time basis of the uploaded data is converted to $\mathrm{BJD}_{\mathrm{TDB}}$. This step is necessary as the observers are allowed to upload their data in any available time basis. However, we recommend JDUTC to be used, as the conversions applied by different software may be subject to assumptions that are not reversible. Also, the data are converted from magnitude to relative flux, if necessary. If the uploaded data do not include uncertainties, these are estimated with a moving standard deviation. In both cases, we perform a first fitting, and then we scale the uncertainties to match the standard deviation of the first fitting residuals. Then, the fitting process is repeated. This is a standard process in the literature applied to observations of HST [28]. In this way, we take into account any extra scatter in the observation and end up with a conservative estimation of the uncertainties in the final results.

The limb darkening coefficients are calculated based on the stellar parameters (temperature, gravity and metallicity) for the planet as provided in the ECC, the Phoenix 3D stellar models and the response curves of the different filters, through the open source Python package ExoTETHyS ${ }^{9}$ [29]. For all the models, we use the 4-coefficients law by Claret 2000 [30].

During the fitting, we fix all the transit parameters, with the exception of the planet-to-star radius ratio and the mid-time, to the values in the ECC. Simultaneously with the transit model, we use a model for the out-of-transit systematics, which are usually due to airmass variations. We consider three models for the systematics (linear with airmass, linear with time, and quadratic with time) and choose the one which best describes the data (minimum reduced chi-square and residuals autocorrelation), at the same time, giving a result for the planet-to-star radius ratio close to the expected value from ECC. We need to note here that all the models used are exposure-time

\footnotetext{
${ }^{8}$ https://github.com/ucl-exoplanets/pylightcurve

${ }^{9}$ https://github.com/ucl-exoplanets/ExoTETHyS
} 
integrated - i.e. they do take into account the variation in the light curve during the exposure. This is achieved by calculating the model for sub-exposures of 10 seconds and then using the average.

The results from each individual light curve fitting are compared with both the literature and other observations on ExoClock. Based on the information from these sources, each observation is either validated by the platform, or returned to the user for further reduction and photometry.

\subsection{Educational material}

Continuous training and interaction between the participants and the science team are fundamental elements to ensure high quality in the produced results. The learning process is complex and multi-dimensional and every person learns in a different way. For these reasons, it is important to support the learning process through a variety of material: manuals; guidelines; videos; podcasts; newsletters; one-to-one feedback; training datasets.

Because our project is open to people from various countries, it is significant to facilitate their participation and avoid misconceptions. Thus, a large part of the educational material is also provided in different languages (at the moment English, Greek, Portuguese, and French) and we also have national contact points for several countries, to assist at a local level. All this material is hosted on a separate website, especially built for educational purposes. ${ }^{10}$

\section{Results - first data release}

Here we present the first data release of the ExoClock project, which includes three data products:

- $\quad$ the Exoplanet Characterisation Catalogue (see Section 2.2)

- the catalogue of ExoClock observations

- the catalogue of ExoClock ephemerides

All data products and their descriptions can found through the OSF repository with DOI: 10.17605/OSF.IO/3W7HM (The repository will become available upon acceptance of this manuscript.)

\subsection{Catalogue of ExoClock observations}

Here we present the first data release of the ExoClock project, containing 632 observations of 120 planets in the ECC (30\%). These observations were conducted between 2011 and 2020, submitted to the ExoClock platform before the $15^{\text {th }}$ of April 2020, and validated according to the criteria described in Section 4.3. The current rate of submitting new observations is above 100 per month. From the 632 observations

\footnotetext{
${ }^{10}$ https://www.exoworldsspies.com
} 
in this dataset, 402 (17\% from amateur astronomers) were acquired before the kick off of the project in September 2019, while the remaining 230 (73\% from amateur astronomers) after September 2019. The largest contribution to this dataset has been made by the Observatoire des Baronnies Provençales with 356 observations, most of which were acquired before September 2019.

Note that in this work, only the observations that were submitted up to the $15^{\text {th }}$ of April 2020 are included. At the moment, the ExoClock platform contains more than 1500 observations (62\% from amateur astronomers), which will be analysed in future publications. In addition, the ECC will be dynamically updated in the future, as more planets that are accessible by Ariel will be discovered. Figure 1 shows the cumulative distribution of all the observations based on the time of observation (not the time of submission).

The online catalogue of ExoClock observations includes the following information for each observation:

1. the raw light curve;

2. metadata regarding the observer(s), the planet observed (link to ECC), the equipment used (telescope-camera-filter), the exposure time and the time and flux formats;

3. the raw light curve filtered for outliers, converted to $\mathrm{BJD}_{\mathrm{TDB}}$ and flux formats and enhanced with the estimation for the uncertainties, the target altitude, and the airmass;

4. the fitting results, including the de-trending method used and its parameters;

5. the de-trended light curve, enhanced with the de-trending model, the transit model and the residuals;

6. fitting diagnostics on the residuals (standard deviation, chi-squared, autocorrelation)

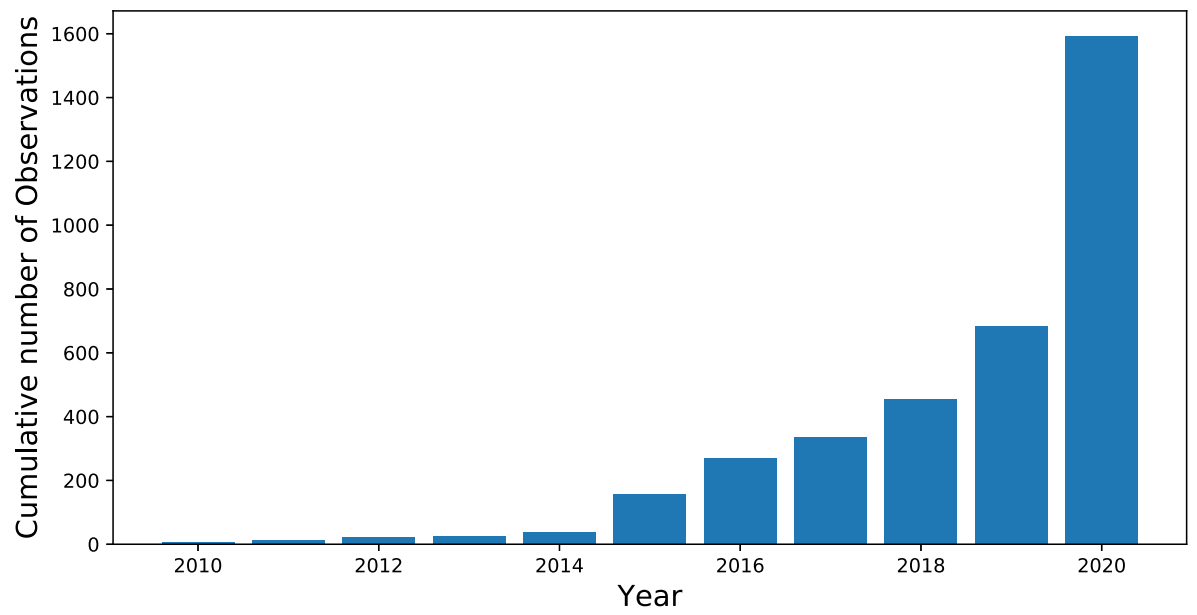

Fig. 1 Cumulative distribution of all the observations in the ExoClock platform, based on the observing date 


\subsection{Catalogue of ExoClock ephemerides}

The ExoClock project aims to deliver a catalogue of verified ephemerides for the planets in the Ariel target list, before the launch of the mission. An ephemeris is considered verified if it gives predictions for the end of 2028 with an uncertainty lower than 1/12 of the transit duration (see Section 2.3). This catalogue is initiated here with verified ephemerides for 84 planets in the ECC (22\%). The verified status of these ephemerides is clearly indicated on the website, to encourage their use by the community. The 84 ephemerides that have been verified will be continuously monitored, as biases in measurements may still exist but require more observations to become statistically significant, and their status will be revised with future data releases.

To produce this catalogue we assessed the ephemerides of the 120 planets observed in this first data release as follows:

1. We updated the ephemerides that did not meet the verification criterion using both the ExoClock observations and values from the literature (19 in total). Note that for some of these planets, data existed in the literature but the ephemerides were either not updated by the authors or the updates where not properly listed in other catalogues. This proves the need for an organised way of listing the ephemeris information for all planets.

2. We evaluated the O-C drift for the remaining ephemerides and identified those for which our observations suggested an important, unexpected, drift (9 in total). An important drift is one that predicts an O-C greater than 1/12 of the transit duration for the end of 2028. We then updated these ephemerides using both the ExoClock observations and values from the literature. Note that these planets met the verification criterion, and the drifts observed were due to biases in the initial ephemerides. This proves the need for continuous and regular follow-up after the transit discovery.

3. We verified the current ephemerides for the planets that were observed at least twice and did not show any significant drift (56 in total). The majority of the planets in this category were marked as low priority.

4. The remaining 36 ephemerides were not verified with the data in this release as only one observation was acquired.

For planets in categories 1 and 2, where the ephemerides updates were vital for continuing monitoring the planets in the future, we did use single observations in some cases (KELT-15b, WASP-13b, WASP-31b, WASP-67b, KELT-7b, WASP-16b). In these cases, there was the need for the update and also there were data, either in the literature or on our website, supporting the observed drift. To update the ephemerides we considered both literature mid-times and the mid-times that resulted from analysing the ExoClock observations. At first, we computed a new zero-epoch as the weighed average of the epochs of the available data.

For the 28 ephemerides that have been updated, we refer the reader to the online data repository for all the data used for the refinement. Figure 2 show the all the 

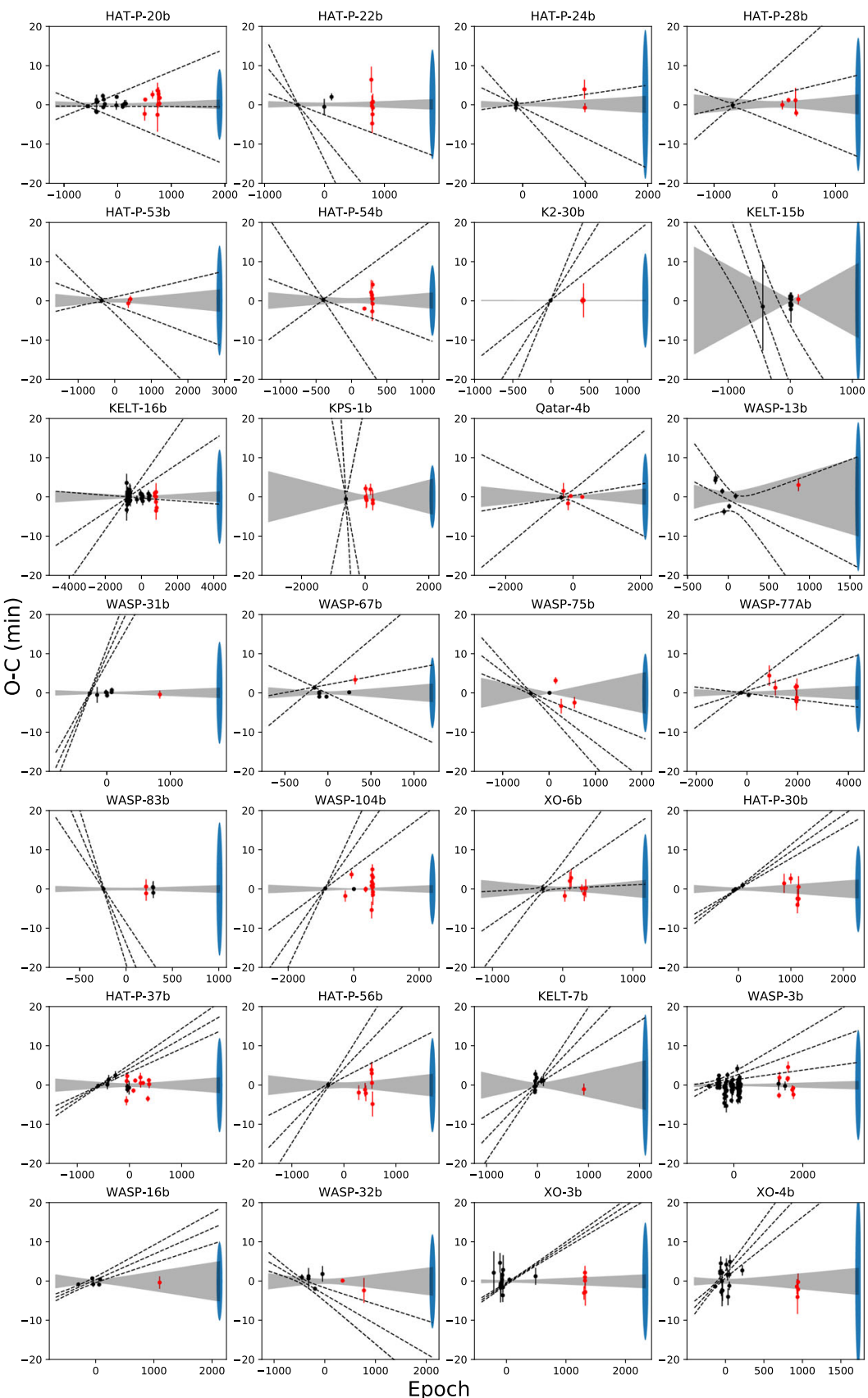

Fig. 2 Comparison between the initial (dashed lines) and the updated ephemerides (shaded area) for the 28 planets that had their ephemerides refined. The black circles indicate literature values while the red ones indicating observations provided by telescopes registered in the ExoClock network. In blue, we indicate the target $1 \sigma$ uncertainty for the end of 2028 for each planet. For all planets, the horizontal axis represents epochs between 01/01/2005 and 01/01/2029 

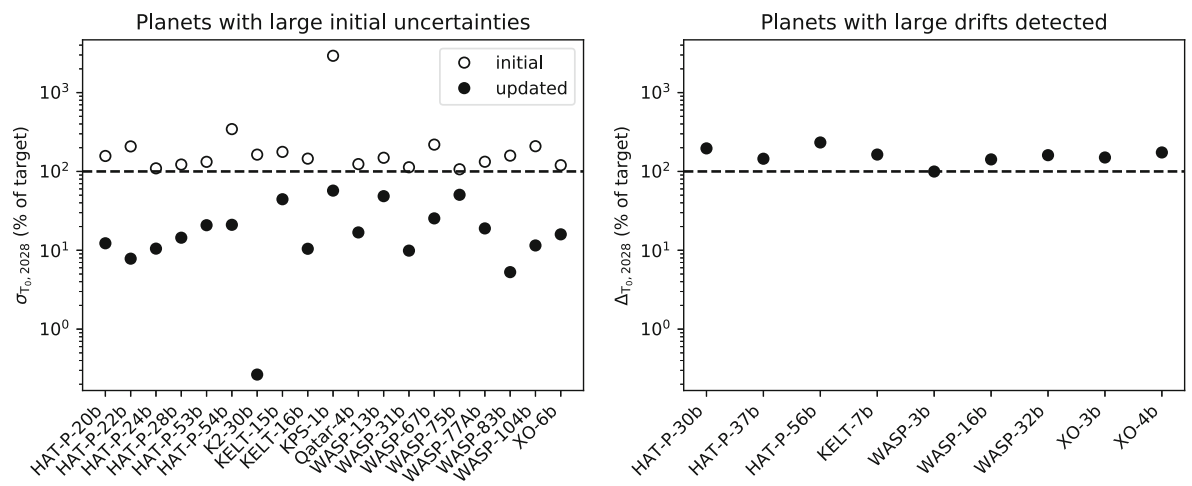

Fig. 3 Left: Comparison between the initial and the updated uncertainties in predictions for the end of 2028 , relatively to the target uncertainty (1/12 of the transit duration) for updated planets in the category 1. Right: Drift of the mid-time predictions for the end of 2028 between the initial and the updated ephemerides for those planets which were found to have unexpected drifts

28 refined ephemerides compared with the initial ones while, Figure 3 shows the variations in the precision and the 2028 predictions for the refined ephemerides. In addition, Table 1 presents the ephemerides that have been updated (categories 1 and 2 ), Table 2 presents the ephemerides that have been verified (category 3 ), and finally Table 3 gives the references for the parameters used in the analysis of all the planets in this data release.

\section{Discussion}

The results presented here demonstrate that a manifold platform like ExoClock is very efficient in making the best use of all available resources towards addressing a defined scientific problem. In our case, the defined problem was the need for upto-date ephemerides in order to increase the efficiency of the Ariel observations and other follow-up activities. However, the project architecture (priorities, organisation, website features etc) could support other endeavours, either in the same field or other sectors, to optimise data collection and ensure reliability and homogeneity in the results.

The open nature of the ExoClock project guarantees the inclusion of various audiences that have the opportunity to contribute directly. This is fundamental to achieve the best use of all currently available resources. Currently, there are 140 participants in ExoClock, of which $80 \%$ are amateur astronomers and the rest include both early career and senior professional astronomers, but also a few students and teachers. These various groups of people come from different backgrounds and have different interests. This is significant since it results in a dynamic team of people who continuously bring new ideas and enrich the complexion of the project. Since also 
Table 1 New ephemerides for the 28 planets that have been updated here (categories 1 and 2), and references for the literature data used

\begin{tabular}{|c|c|c|c|}
\hline Planet & New $\mathrm{T}_{0}\left(\mathrm{BJD}_{\mathrm{TDB}}\right)$ & New Period (days) & $\begin{array}{l}\text { References for } \\
\text { literature data used }\end{array}$ \\
\hline HAT-P-20b & $2456670.97803 \pm 0.0001$ & $2.875317 \pm 4 \mathrm{e}-07$ & [36-39] \\
\hline HAT-P-22b & $2456366.08903 \pm 0.00025$ & $3.212233 \pm 4 \mathrm{e}-07$ & {$[36,40]$} \\
\hline HAT-P-24b & $2455592.76491 \pm 0.00023$ & $3.3552454 \pm 7 \mathrm{e}-07$ & {$[41]$} \\
\hline HAT-P-28b & $2457713.934 \pm 0.0005$ & $3.2572124 \pm 1.2 \mathrm{e}-06$ & {$[42]$} \\
\hline HAT-P-53b & $2456514.0562 \pm 0.00025$ & $1.9616265 \pm 7 \mathrm{e}-07$ & {$[43]$} \\
\hline HAT-P-54b & $2457811.6454 \pm 0.0004$ & $3.7998518 \pm 1.1 \mathrm{e}-06$ & {$[44]$} \\
\hline $\mathrm{K} 2-30 \mathrm{~b}$ & $2457112.988874 \pm 1.3 \mathrm{e}-06$ & $4.098481163 \pm 1.8 \mathrm{e}-08$ & {$[45]$} \\
\hline KELT-15b & $2458544.07707 \pm 0.00018$ & $3.329472 \pm 6 \mathrm{e}-06$ & {$[46,47]$} \\
\hline KELT-16b & $2457946.86088 \pm 0.0001$ & $0.96899295 \pm 2 \mathrm{e}-07$ & {$[48-50]$} \\
\hline KPS-1b & $2458544.108 \pm 0.0003$ & $1.7063219 \pm 1.5 \mathrm{e}-06$ & {$[51]$} \\
\hline Qatar-4b & $2458293.1227 \pm 0.00015$ & $1.805369 \pm 6 \mathrm{e}-07$ & {$[52]$} \\
\hline WASP-13b & $2455218.5681 \pm 0.0008$ & $4.353019 \pm 4 \mathrm{e}-06$ & {$[53,54]$} \\
\hline WASP-31b & $2456088.4374 \pm 0.0001$ & $3.4058853 \pm 5 \mathrm{e}-07$ & {$[55-57]$} \\
\hline WASP-67b & $2456548.83747 \pm 0.0002$ & $4.6144172 \pm 1.3 \mathrm{e}-06$ & {$[58-60]$} \\
\hline WASP-75b & $2457007.46252 \pm 5 \mathrm{e}-05$ & $2.4841993 \pm 1.7 \mathrm{e}-06$ & {$[61,62]$} \\
\hline WASP-77Ab & $2456224.05817 \pm 0.00017$ & $1.3600295 \pm 3 \mathrm{e}-07$ & {$[40,63]$} \\
\hline WASP-104b & $2457928.048611 \pm 1.7 \mathrm{e}-05$ & $1.7554057 \pm 3 \mathrm{e}-07$ & {$[65,66]$} \\
\hline $\mathrm{XO}-6 \mathrm{~b}$ & $2457737.031 \pm 0.0003$ & $3.7649924 \pm 1.3 \mathrm{e}-06$ & {$[67]$} \\
\hline HAT-P-30b & $2455765.63288 \pm 0.00018$ & $2.8106019 \pm 7 \mathrm{e}-07$ & {$[68-70]$} \\
\hline HAT-P-37b & $2457303.82448 \pm 0.00024$ & $2.7974418 \pm 7 \mathrm{e}-07$ & {$[70-72]$} \\
\hline HAT-P-56b & $2457404.8183 \pm 0.0004$ & $2.7908233 \pm 1.1 \mathrm{e}-06$ & {$[73]$} \\
\hline KELT-7b & $2456385.3121 \pm 0.0004$ & $2.7347694 \pm 2 \mathrm{e}-06$ & {$[74]$} \\
\hline WASP-3b & $2455517.89652 \pm 0.0001$ & $1.84683506 \pm 1.8 \mathrm{e}-07$ & {$[75-85]$} \\
\hline WASP-16b & $2455495.0619 \pm 0.0003$ & $3.1186023 \pm 1.6 \mathrm{e}-06$ & {$[86,87]$} \\
\hline WASP-32b & $2456379.8909 \pm 0.0004$ & $2.7186643 \pm 1.1 \mathrm{e}-06$ & {$[80,88,89]$} \\
\hline $\mathrm{XO}-3 \mathrm{~b}$ & $2454721.14865 \pm 0.00014$ & $3.191526 \pm 5 \mathrm{e}-07$ & {$[72,90-94]$} \\
\hline $\mathrm{XO}-4 \mathrm{~b}$ & $2455067.5685 \pm 0.0003$ & $4.1250683 \pm 1.3 \mathrm{e}-06$ & [95-98] \\
\hline
\end{tabular}

ExoClock observers have different levels of experience, they contribute to the project from different perspectives.

Through the course of the project, it became clear that an integrated platform like ExoClock is beneficial to the whole exoplanet community as it can support maximising the efficiency of any organised observing effort and maintaining an always up-to-date scientific product. This is done through providing a uniformly 
Table 2 Verified literature ephemerides for the 56 planets in category 3

\begin{tabular}{|c|c|c|c|}
\hline Planet & $\mathrm{T}_{0}\left(\mathrm{BJD}_{\mathrm{TDB}}\right)$ & Period (days) & Reference \\
\hline CoRoT-2b & $2454237.53616 \pm 3 \mathrm{e}-05$ & $1.74299609 \pm 2 \mathrm{e}-07$ & [99] \\
\hline GJ1214b & $2454934.9177 \pm 3 \mathrm{e}-05$ & $1.58040494 \pm 9 \mathrm{e}-08$ & {$[100]$} \\
\hline GJ436b & $2454865.08403 \pm 3 e-05$ & $2.643898 \pm 3 \mathrm{e}-07$ & {$[101]$} \\
\hline HAT-P-1b & $2453979.93277 \pm 0.00024$ & $4.4652998 \pm 6 e-07$ & [102] \\
\hline HAT-P-3b & $2454856.702 \pm 0.0001$ & $2.899736 \pm 2 \mathrm{e}-06$ & [103] \\
\hline HAT-P-4b & $2454245.8152 \pm 0.0002$ & $3.0565254 \pm 1.2 \mathrm{e}-06$ & [80] \\
\hline HAT-P-5b & $2455432.4551 \pm 0.0001$ & $2.7884736 \pm 5 \mathrm{e}-07$ & {$[104]$} \\
\hline HAT-P-9b & $2455484.9131 \pm 0.0004$ & $3.9228107 \pm 1 \mathrm{e}-06$ & {$[105]$} \\
\hline HAT-P-12b & $2454419.19584 \pm 9 \mathrm{e}-05$ & $3.2130589 \pm 3 \mathrm{e}-07$ & {$[106]$} \\
\hline HAT-P-13b & $2455176.53893 \pm 0.00022$ & $2.9162433 \pm 1.2 \mathrm{e}-06$ & {$[106]$} \\
\hline HAT-P-16b & $2455027.59292 \pm 0.00019$ & $2.7759704 \pm 7 \mathrm{e}-07$ & {$[106]$} \\
\hline HAT-P-19b & $2455091.535 \pm 0.00015$ & $4.0087842 \pm 7 \mathrm{e}-07$ & {$[107]$} \\
\hline HAT-P-23b & $2454852.26548 \pm 0.00017$ & $1.2128867 \pm 2 \mathrm{e}-07$ & {$[106]$} \\
\hline HAT-P-25b & $2457006.91299 \pm 0.00021$ & $3.6528159 \pm 7 \mathrm{e}-07$ & {$[1]$} \\
\hline HAT-P-32b & $2454420.44712 \pm 9 \mathrm{e}-05$ & $2.150008 \pm 1 \mathrm{e}-06$ & [108] \\
\hline HAT-P-36b & $2455565.1816 \pm 0.0004$ & $1.3273468 \pm 5 \mathrm{e}-07$ & {$[109]$} \\
\hline HAT-P-44b & $2456204.47794 \pm 0.00019$ & $4.3011886 \pm 1 \mathrm{e}-06$ & {$[1]$} \\
\hline HAT-P-51b & $2456194.1228 \pm 0.0004$ & $4.218028 \pm 6 \mathrm{e}-06$ & {$[43]$} \\
\hline HAT-P-52b & $2456645.1398 \pm 0.0003$ & $2.7535965 \pm 1.1 \mathrm{e}-06$ & {$[1]$} \\
\hline HD189733b & $2454279.437468 \pm 1.5 \mathrm{e}-05$ & $2.21857567 \pm 1.5 \mathrm{e}-07$ & [110] \\
\hline KELT-1b & $2457306.976 \pm 0.0003$ & $1.2174928 \pm 6 \mathrm{e}-07$ & {$[111]$} \\
\hline KELT-3b & $2456269.4899 \pm 0.0003$ & $2.703385 \pm 1.8 \mathrm{e}-06$ & {$[1]$} \\
\hline Kepler-6b & $2454954.48742 \pm 3 \mathrm{e}-05$ & $3.23469918 \pm 3 \mathrm{e}-08$ & [112] \\
\hline Qatar-1b & $2456234.10322 \pm 6 \mathrm{e}-05$ & $1.4200242 \pm 2.2 \mathrm{e}-07$ & [113] \\
\hline Qatar-2b & $2457250.201605 \pm 8 \mathrm{e}-06$ & $1.33711677 \pm 1 \mathrm{e}-07$ & [114] \\
\hline TrES-1b & $2453186.807 \pm 0.00012$ & $3.030072 \pm 3 \mathrm{e}-07$ & {$[80]$} \\
\hline TrES-2b & $2454955.764103 \pm 6 \mathrm{e}-06$ & $2.47061338 \pm 1 \mathrm{e}-08$ & {$[112]$} \\
\hline TrES-3b & $2454185.91116 \pm 6 \mathrm{e}-05$ & $1.30618619 \pm 1.5 \mathrm{e}-07$ & {$[115]$} \\
\hline TrES-4b & $2454230.9056 \pm 0.0003$ & $3.5539277 \pm 5 \mathrm{e}-07$ & [116] \\
\hline TrES-5b & $2455443.25271 \pm 0.00011$ & $1.48224754 \pm 1.7 \mathrm{e}-07$ & {$[70]$} \\
\hline WASP-1b & $2453912.5151 \pm 0.0003$ & $2.5199454 \pm 5 \mathrm{e}-07$ & {$[117]$} \\
\hline WASP-2b & $2453991.51536 \pm 0.00018$ & $2.1522213 \pm 4 \mathrm{e}-07$ & {$[80]$} \\
\hline WASP-10b & $2454664.03804 \pm 6 \mathrm{e}-05$ & $3.0927295 \pm 3 \mathrm{e}-07$ & {$[106]$} \\
\hline WASP-11b & $2454729.9072 \pm 0.0002$ & $3.7224797 \pm 4 \mathrm{e}-07$ & [109] \\
\hline WASP-12b & $2456176.66826 \pm 8 \mathrm{e}-05$ & $1.0914203 \pm 1.4 \mathrm{e}-07$ & [113] \\
\hline WASP-19b & $2455708.534626 \pm 1.9 \mathrm{e}-05$ & $0.78883899 \pm 4 \mathrm{e}-08$ & {$[118]$} \\
\hline WASP-24b & $2455687.75616 \pm 0.00016$ & $2.3412217 \pm 8 \mathrm{e}-07$ & [119] \\
\hline WASP-36b & $2455569.8377 \pm 0.0005$ & $1.53736596 \pm 2.4 \mathrm{e}-07$ & {$[120]$} \\
\hline WASP-43b & $2455528.86863 \pm 5 \mathrm{e}-05$ & $0.81347398 \pm 4 \mathrm{e}-08$ & {$[121]$} \\
\hline WASP-48b & $2455364.55241 \pm 0.00024$ & $2.1436354 \pm 6 \mathrm{e}-07$ & {$[122]$} \\
\hline WASP-49b & $2456267.6839 \pm 0.00013$ & $2.7817362 \pm 1.4 \mathrm{e}-06$ & {$[123]$} \\
\hline WASP-50b & $2455558.6124 \pm 0.0002$ & $1.9550938 \pm 1.3 \mathrm{e}-06$ & {$[124]$} \\
\hline
\end{tabular}


Table 2 (continued)

\begin{tabular}{llll}
\hline Planet & $\mathrm{T}_{0}\left(\mathrm{BJD}_{\mathrm{TDB}}\right)$ & Period (days) & Reference \\
\hline WASP-52b & $2456862.79795 \pm 7 \mathrm{e}-05$ & $1.74978114 \pm 1.4 \mathrm{e}-07$ & {$[125]$} \\
WASP-57b & $2456058.5491 \pm 0.00016$ & $2.8389186 \pm 8 \mathrm{e}-07$ & {$[126]$} \\
WASP-65b & $2456110.68772 \pm 0.00015$ & $2.3114243 \pm 1.5 \mathrm{e}-06$ & {$[61]$} \\
WASP-80b & $2456487.42578 \pm 3 \mathrm{e}-05$ & $3.0678523 \pm 8 \mathrm{e}-07$ & {$[127]$} \\
WASP-85Ab & $2456847.473634 \pm 1.4 \mathrm{e}-05$ & $2.6556777 \pm 4 \mathrm{e}-07$ & {$[128]$} \\
WASP-92b & $2456381.2842 \pm 0.0003$ & $2.1746742 \pm 1.6 \mathrm{e}-06$ & {$[129]$} \\
WASP-93b & $2456079.565 \pm 0.0004$ & $2.7325321 \pm 2 \mathrm{e}-06$ & {$[129]$} \\
WASP-103b & $2456836.29644 \pm 6 \mathrm{e}-05$ & $0.9255456 \pm 1.3 \mathrm{e}-06$ & {$[130]$} \\
WASP-113b & $2457197.09823 \pm 4 \mathrm{e}-05$ & $4.542169 \pm 4 \mathrm{e}-06$ & {$[131]$} \\
WASP-114b & $2456667.73661 \pm 0.00021$ & $1.5487743 \pm 1.2 \mathrm{e}-06$ & {$[131]$} \\
WASP-153b & $2453142.543 \pm 0.003$ & $3.332609 \pm 2 \mathrm{e}-06$ & {$[132]$} \\
XO-1b & $2453887.74774 \pm 0.00022$ & $3.9415069 \pm 1.8 \mathrm{e}-06$ & {$[133]$} \\
XO-2Nb & $2455565.54648 \pm 5 \mathrm{e}-05$ & $2.6158592 \pm 3 \mathrm{e}-07$ & {$[134]$} \\
XO-5b & $2456864.3137 \pm 0.0002$ & $4.1877558 \pm 6 \mathrm{e}-07$ & {$[135]$} \\
\hline
\end{tabular}

derived reference catalogue, allocating observations according to the capabilities of the observatories (personal schedule), and prioritising the observations.

One of the most important elements in the ExoClock platform is the alert system, which was developed especially for planets that showed unexpected drifts. This system proved very effective so far, with the participants being very responsive to such alerts. Apart from large O-C drifts, the alert system helped us identify mistakes in the initial catalogues, related to either the ephemerides or the transit parameters. An example was the case of WASP-84b, for which the initial ephemeris was mistyped in the NASA Exoplanet archive, leading to wrong transit predictions by many hours. Similarly, a mistyped value for Kepler-447b led to a much larger transit depth, a mistake identified after observations made by the ExoClock participants. These results would not had be identified and cross-validated without the participation of an active community that is always willing to observe, even planets with low or medium priority.

Certainly, the highly interactive design of the ExoClock platform is another key element, and it is achieved through various approaches, such as: educational material, training, personal feedback, meeting, newsletters, and, even more importantly, the development of user friendly tools that enable participation from inexperienced observers. This is an element that contributes significantly towards achieving homogeneity in the results, as it supports the efficient implementation of the data collection and analysis protocols described above. It is important to note that $70 \%$ of the ExoClock participants use HOPS for data reduction and photometry. 
Table 3 Sources for the parameters used in the analysis of all 120 planets in this data release

\begin{tabular}{|c|c|c|c|c|c|}
\hline CoRoT-2b & {$[99,136,137]$} & HAT-P-65b & {$[138,138,138]$} & WASP-26b & {$[119,119,139]$} \\
\hline GJ1214b & {$[100,140,141]$} & HATS-6b & {$[142,142,142]$} & WASP-31b & {$[55,55,55]$} \\
\hline GJ436b & {$[101,143,144]$} & HATS-22b & {$[145,145,145]$} & WASP-32b & {$[80,88,88]$} \\
\hline HAT-P-1b & {$[102,102,143]$} & HATS-25b & {$[146,146,146]$} & WASP-36b & {$[120,120,147]$} \\
\hline HAT-P-3b & {$[103,103,143]$} & HD189733b & {$[110,143,148]$} & WASP-43b & {$[121,121,149]$} \\
\hline HAT-P-4b & {$[78,80,143]$} & HD209458b & {$[143,143,150]$} & WASP-48b & {$[69,122,122]$} \\
\hline HAT-P-5b & {$[104,143,143]$} & HD80606b & {$[151,152,152]$} & WASP-49b & {$[123,123,153]$} \\
\hline HAT-P-6b & {$[143,154,154]$} & $\mathrm{K} 2-29 \mathrm{~b}$ & {$[155,155,155]$} & WASP-50b & {$[124,156,156]$} \\
\hline HAT-P-7b & {$[112,118,157]$} & $\mathrm{K} 2-30 \mathrm{~b}$ & {$[45,45,45]$} & WASP-52b & {$[125,158,158]$} \\
\hline HAT-P-8b & {$[159,160,160]$} & KELT-1b & {$[111,111,161]$} & WASP-55b & {$[58,162,162]$} \\
\hline HAT-P-9b & {$[105,105,163]$} & KELT-3b & {$[1,164,164]$} & WASP-57b & {$[126,126,165]$} \\
\hline HAT-P-12b & {$[106,166,166]$} & KELT-7b & {$[74,74,74]$} & WASP-58b & {$[1,158,158]$} \\
\hline HAT-P-13b & {$[106,167,167]$} & KELT-8b & {$[1,168,168]$} & WASP-65b & {$[61,61,61]$} \\
\hline HAT-P-16b & {$[106,169,169]$} & KELT-15b & {$[46,46,46]$} & WASP-67b & {$[58,58,58]$} \\
\hline HAT-P-17b & {$[170,170,170]$} & KELT-16b & {$[48,48,48]$} & WASP-69b & {$[171,171,171]$} \\
\hline HAT-P-18b & {$[107,172,173]$} & KPS-1b & {$[51,51,51]$} & WASP-74b & {$[64,64,64]$} \\
\hline HAT-P-19b & {$[107,172,172]$} & Kepler-6b & {$[112,174,175]$} & WASP-75b & {$[61,61,61]$} \\
\hline HAT-P-20b & {$[36,36,36]$} & Qatar-1b & {$[113,113,176]$} & WASP-76b & {$[177,177,177]$} \\
\hline HAT-P-22b & {$[36,36,36]$} & Qatar-2b & {$[114,114,178]$} & WASP-77Ab & {$[63,63,63]$} \\
\hline HAT-P-23b & {$[36,106,122]$} & Qatar-3b & {$[1,52,52]$} & WASP-80b & {$[127,127,179]$} \\
\hline HAT-P-24b & {$[41,41,41]$} & Qatar-4b & {$[1,52,52]$} & WASP-82b & {$[135,177,177]$} \\
\hline HAT-P-25b & {$[1,180,181]$} & Qatar-5b & {$[1,52,52]$} & WASP-83b & {$[64,64,64]$} \\
\hline HAT-P-26b & {$[182,182,183]$} & TrES-1b & {$[80,143,143]$} & WASP-84b & {$[171,171,171]$} \\
\hline HAT-P-28b & {$[42,42,42]$} & TrES-2b & {$[112,143,175]$} & WASP- $85 \mathrm{Ab}$ & {$[128,184,184]$} \\
\hline HAT-P-30b & {$[68,70,70]$} & TrES-3b & {$[78,115,143]$} & WASP-92b & {$[129,129,129]$} \\
\hline HAT-P-32b & {$[105,108,108]$} & TrES-4b & {$[116,116,143]$} & WASP-93b & {$[129,129,129]$} \\
\hline HAT-P-36b & {$[71,105,109]$} & TrES-5b & {$[70,70,185]$} & WASP-103b & {$[130,186,187]$} \\
\hline HAT-P-37b & {$[70,70,71]$} & WASP-1b & {$[117,117,143]$} & WASP-104b & {$[65,65,65]$} \\
\hline HAT-P-38b & {$[1,188,188]$} & WASP-2b & {$[80,143,189]$} & WASP-107b & {$[190,191,191]$} \\
\hline HAT-P-39b & {$[192,192,192]$} & WASP-3b & {$[75,78,78]$} & WASP-113b & {$[131,131,131]$} \\
\hline HAT-P-41b & {$[192,192,192]$} & WASP-10b & {$[106,193,194]$} & WASP-114b & {$[131,131,131]$} \\
\hline HAT-P-44b & {$[1,195,195]$} & WASP-11b & {$[109,196,197]$} & WASP-127b & {$[198,198,199]$} \\
\hline HAT-P-46b & {$[1,195,195]$} & WASP-12b & {$[113,113,200]$} & WASP-153b & {$[132,132,132]$} \\
\hline HAT-P-49b & {$[201,201,201]$} & WASP-13b & {$[53,53,54]$} & WASP-167b & {$[202,202,202]$} \\
\hline HAT-P-51b & {$[43,43,43]$} & WASP-15b & {$[87,87,203]$} & $\mathrm{XO}-1 \mathrm{~b}$ & {$[133,143,143]$} \\
\hline HAT-P-52b & {$[1,43,43]$} & WASP-16b & {$[86,87,87]$} & $\mathrm{XO}-2 \mathrm{Nb}$ & {$[134,134,143]$} \\
\hline HAT-P-53b & {$[43,43,43]$} & WASP-19b & {$[118,118,204]$} & $\mathrm{XO}-3 \mathrm{~b}$ & {$[90,205,205]$} \\
\hline HAT-P-54b & {$[44,44,44]$} & WASP-21b & {$[107,206,206]$} & $\mathrm{XO}-4 \mathrm{~b}$ & {$[95,96,96]$} \\
\hline HAT-P-56b & {$[73,73,73]$} & WASP-24b & {$[119,119,207]$} & $\mathrm{XO}-5 \mathrm{~b}$ & {$[135,135,208]$} \\
\hline HAT-P-57b & {$[209,209,209]$} & WASP-25b & {$[119,119,210]$} & XO-6b & {$[67,67,67]$} \\
\hline
\end{tabular}

For each planet, three references are provided, one for the stellar parameters used to calculate the limb darkening, one for the transit parameters and one for the initial ephemeris (before any update) 


\section{Conclusions}

From the experience gained in ExoClock, we identified the importance of making the best use of all available resources through organising community-wide efforts. This is a prototype that can be used in other fields apart from exoplanets. Organising a project in a collaborative perspective by considering contributions from various audiences can maximise the outcome of the available resources. To implement efficiently such a project it is vital to create data collection and validation protocols, educational material (guides, newsletters) and easy-to-use interfaces (user-friendly software and website). In future studies, we will provide the ephemerides verification and updates for more Ariel targets according to new discoveries. The monitoring of the Ariel candidate targets will be continued until the launch of the mission but ExoClock can be established as a platform and used by the exoplanet communities for further purposes. The open framework of ExoClock is vital to facilitate future exoplanet research and avoid wasting resources.

Acknowledgments This project has received funding from the European Research Council (ERC) under the European Union's Horizon 2020 research and innovation programme (grant agreement No 758892, ExoAI). Furthermore, we acknowledge funding by the Science and Technology Funding Council (STFC) grants: ST/K502406/1, ST/P000282/1, ST/P002153/1, and ST/S002634/1. L.V. M is supported by ASI grant n. 2018.22.HH.O. P. Pintr acknowledges the support by the project No.CZ.02.1.01/0.0/0.0/16_026/0008390.

\section{Declarations}

Conflict of interests The authors declare that they have no conflict of interest.

Software and data Software used: Django, PyLightcurve [27], ExoTETHyS [29], Astropy [31], emcee [32], Matplotlib [33], Numpy [34], SciPy [35].

All the data products and their descriptions can found through the OSF repository with DOI: 10.17605/OSF.IO/3W7HM.

Open Access This article is licensed under a Creative Commons Attribution 4.0 International License, which permits use, sharing, adaptation, distribution and reproduction in any medium or format, as long as you give appropriate credit to the original author(s) and the source, provide a link to the Creative Commons licence, and indicate if changes were made. The images or other third party material in this article are included in the article's Creative Commons licence, unless indicated otherwise in a credit line to the material. If material is not included in the article's Creative Commons licence and your intended use is not permitted by statutory regulation or exceeds the permitted use, you will need to obtain permission directly from the copyright holder. To view a copy of this licence, visit http://creativecommonshorg/licenses/by/4. $0 \%$.

\section{References}

1. Mallonn, M., von Essen, C., Herrero, E., Alexoudi, X., Granzer, T., Sosa, M., Strassmeier, K.G., Bakos, G., Bayliss, D., Brahm, R., Bretton, M., Campos, F., Carone, L., Colón, K.D., Dale, H.A., Dragomir, D., Espinoza, N., Evans, P., Garcia, F., Gu, S.H., Guerra, P., Jongen, Y., Jordán, A., Kang, W., Keles, E., Kim, T., Lendl, M., Molina, D., Salisbury, M., Scaggiante, F., Shporer, A., Siverd, R., Sokov, E., Sokova, I., Wünsche, A.: Ephemeris refinement of 21 
hot Jupiter exoplanets with high timing uncertainties. Astron. Astrophys. 622, A81 (2019). https://doi.org/10.1051/0004-6361/201834194

2. Dragomir, D., Harris, M., Pepper, J., Barclay, T., Villanueva, J., Steven, Ricker, G.R., Vanderspek, R., Latham, D.W., Seager, S., Winn, J.N., Jenkins, J.M., Ciardi, D.R., Furesz, G., Henze, C.E., Mireles, I., Morgan, E.H., Quintana, E.V., Ting, E.B., Yahalomi, D.: Securing the legacy of TESS through the care and maintenance of TESS planet ephemerides. AJ 159(5), 219 (2020). https://doi.org/10.3847/1538-3881/ab845d

3. Zellem, R.T., Pearson, K.A., Blaser, E., Fowler, M., Ciardi, D.R., Biferno, A., Massey, B., Marchis, F., Baer, R., Ball, C., Chasin, M., Conley, M., Dixon, S., Fletcher, E., Hernandez, S., Nair, S., Perian, Q., Sienkiewicz, F., Tock, K., Vijayakumar, V., Swain, M.R., Roudier, G.M., Bryden, G., Conti, D.M., Hill, D.H., Hergenrother, C.W., Dussault, M., Kane, S.R., Fitzgerald, M., Boyce, P., Peticolas, L., Gee, W., Cominsky, L., Zimmerman-Brachman, R., Smith, D., Creech-Eakman, M.J., Engelke, J., Iturralde, A., Dragomir, D., Jovanovic, N., Lawton, B., Arbouch, E., Kuchner, M., Malvache, A.: Utilizing small telescopes operated by citizen scientists for transiting exoplanet follow-up. Publ. Astron. Soc. Pac. 132(1011), 054401 (2020). https://doi.org/10.1088/1538-3873/ab7ee7

4. Alonso, R., Moutou, C., Endl, M., Almenara, J.M., Guenther, E.W., Deleuil, M., Hatzes, A., Aigrain, S., Auvergne, M., Baglin, A., Barge, P., Bonomo, A.S., Bordé, P., Bouchy, F., Cavarroc, C., Cabrera, J., Carpano, S., Csizmadia, S., Cochran, W.D., Deeg, H.J., Díaz, R.F., Dvorak, R., Erikson, A., Ferraz-Mello, S., Fridlund, M., Fruth, T., Gandolfi, D., Gillon, M., Grziwa, S., Guillot, T., Hébrard, G., Jorda, L., Léger, A., Lammer, H., Lovis, C., MacQueen, P.J., Mazeh, T., Ofir, A., Ollivier, M., Pasternacki, T., Pätzold, M., Queloz, D., Rauer, H., Rouan, D., Santerne, A., Schneider, J., Tadeu dos Santos, M., Tingley, B., Titz-Weider, R., Weingrill, J., Wuchterl, G.: Transiting exoplanets from the CoRoT space mission. XXVI. CoRoT-24: a transiting multiplanet system. Astron. Astrophys. 567, A112 (2014). https://doi.org/10.1051/0004-6361/201118662

5. Benneke, B., Werner, M., Petigura, E., Knutson, H., Dressing, C., Crossfield, I.J.M., Schlieder, J.E., Livingston, J., Beichman, C., Christiansen, J., Krick, J., Gorjian, V., Howard, A.W., Sinukoff, E., Ciardi, D.R., Akeson, R.L.: Spitzer observations confirm and rescue the habitable-zone super-earth K2-18b for future characterization. ApJ 834(2), 187 (2017). https://doi.org/10.3847/1538-4357/834/2/187

6. Agol, E., Steffen, J., Sari, R., Clarkson, W.: On detecting terrestrial planets with timing of giant planet transits. MNRAS 359, 567 (2005). https://doi.org/10.1111/j.1365-2966.2005.08922.x

7. Maciejewski, G., Dimitrov, D., Fernández, M., Sota, A., Nowak, G., Ohlert, J., Nikolov, G., Bukowiecki, Ł., Hinse, T.C., Pallé, E., Tingley, B., Kjurkchieva, D., Lee, J.W., Lee, C.U.: Departure from the constant-period ephemeris for the transiting exoplanet WASP-12. Astron. Astrophys. 588, L6 (2016). https://doi.org/10.1051/0004-6361/201628312

8. Bouma, L.G., Winn, J.N., Baxter, C., Bhatti, W., Dai, F., Daylan, T., Désert, J.M., Hill, M.L., Kane, S.R., Stassun, K.G., Villasenor, J., Ricker, G.R., Vanderspek, R., Latham, D.W., Seager, S., Jenkins, J.M., Berta-Thompson, Z., Colón, K., Fausnaugh, M., Glidden, A., Guerrero, N., Rodriguez, J.E., Twicken, J.D., Wohler, B.: WASP-4b arrived early for the TESS mission. AJ 157(6), 217 (2019). https://doi.org/10.3847/1538-3881/ab189f

9. Beck, P., Robson, L., Gallaway, M., Jones, H.R.A., Campbell, D.: Efficient follow-up of exoplanet transits using small telescopes. Publ. Astron. Soc. Pac. 131(1002), 084402 (2019). https://doi.org/10.1088/1538-3873/ab1eb4

10. Edwards, B., Changeat, Q., Yip, K.H., Tsiaras, A., Taylor, J., Akhtar, B., AlDaghir, J., Bhattarai, P., Bhudia, T., Chapagai, A., Huang, M., Kabir, D., Khag, V., Khaliq, S., Khatri, K., Kneth, J., Kothari, M., Najmudin, I., Panchalingam, L., Patel, M., Premachand ran, L., Qayyum, A., Rana, P., Shaikh, Z., Syed, S., Theti, H., Zaidani, M., Saraf, M., de Mijolla, D., Caines, H., Kokori, A., Rocchetto, M., Mallonn, M., Bachschmidt, M., Bosch, J.M., Bretton, M., Chatelain, P., Deldem, M., Sisto, R.D., Evans, P., Fernández-Lajús, E., Guerra, P., Horta, F.G., Kang, W., Kim, T., Leroy, A., Lomoz, F., de Haro, J.L., Hentunen, V.P., Jongen, Y., Molina, D., Montaigut, R., Naves, R., Raetz, M., Sauer, T., Watkins, A., Wünsche, A., Zibar, M., Dunn, W., Tessenyi, M., Savini, G., Tinetti, G., Tennyson, J.: Original research by young twinkle students (ORBYTS): Ephemeris refinement of transiting exoplanets. MNRAS 4(7), 109 (2020). https://doi.org/10.1093/mnras/staa1245

11. Kabáth, P., Žák, J., Boffin, H.M.J., Ivanov, V.D., Jones, D.: Detection limits of exoplanetary atmospheres with 2-m class telescopes. Publ. Astron. Soc. Pac. 131(1002), 085001 (2019). https://doi.org/10.1088/1538-3873/ab2143 
12. Tinetti, G., Drossart, P., Eccleston, P., Hartogh, P., Heske, A., Leconte, J., Micela, G., Ollivier, M., Pilbratt, G., Puig, L., Turrini, D., Vandenbussche, B., Wolkenberg, P., Beaulieu, J.P., Buchave, L.A., Ferus, M., Griffin, M., Guedel, M., Justtanont, K., Lagage, P.O., Machado, P., Malaguti, G., Min, M., Nørgaard-Nielsen, H.U., Rataj, M., Ray, T., Ribas, I., Swain, M., Szabo, R., Werner, S., Barstow, J., Burleigh, M., Cho, J., du Foresto, V.C., Coustenis, A., Decin, L., Encrenaz, T., Galand, M., Gillon, M., Helled, R., Morales, J.C., Muñoz, A.G., Moneti, A., Pagano, I., Pascale, E., Piccioni, G., Pinfield, D., Sarkar, S., Selsis, F., Tennyson, J., Triaud, A., Venot, O., Waldmann, I., Waltham, D., Wright, G., Amiaux, J., Auguères, J.L., Berthé, M., Bezawada, N., Bishop, G., Bowles, N., Coffey, D., Colomé, J., Crook, M., Crouzet, P.E., Da Peppo, V., Sanz, I.E., Focardi, M., Frericks, M., Hunt, T., Kohley, R., Middleton, K., Morgante, G., Ottensamer, R., Pace, E., Pearson, C., Stamper, R., Symonds, K., Rengel, M., Renotte, E., Ade, P., Affer, L., Alard, C., Allard, N., Altieri, F., André, Y., Arena, C., Argyriou, I., Aylward, A., Baccani, C., Bakos, G., Banaszkiewicz, M., Barlow, M., Batista, V., Bellucci, G., Benatti, S., Bernardi, P., Bézard, B., Blecka, M., Bolmont, E., Bonfond, B., Bonito, R., Bonomo, A.S., Brucato, J.R., Brun, A.S., Bryson, I., Bujwan, W., Casewell, S., Charnay, B., Pestellini, C.C., Chen, G., Ciaravella, A., Claudi, R., Clédassou, R., Damasso, M., Damiano, M., Danielski, C., Deroo, P., Di Giorgio, A.M., Dominik, C., Doublier, V., Doyle, S., Doyon, R., Drummond, B., Duong, B., Eales, S., Edwards, B., Farina, M., Flaccomio, E., Fletcher, L., Forget, F., Fossey, S., Fränz, M., Fujii, Y., García-Piquer, Á., Gear, W., Geoffray, H., Gérard, J.C., Gesa, L., Gomez, H., Graczyk, R., Griffith, C., Grodent, D., Guarcello, M.G., Gustin, J., Hamano, K., Hargrave, P., Hello, Y., Heng, K., Herrero, E., Hornstrup, A., Hubert, B., Ida, S., Ikoma, M., Iro, N., Irwin, P., Jarchow, C., Jaubert, J., Jones, H., Julien, Q., Kameda, S., Kerschbaum, F., Kervella, P., Koskinen, T., Krijger, M., Krupp, N., Lafarga, M., Landini, F., Lellouch, E., Leto, G., Luntzer, A., Rank-Lüftinger, T., Maggio, A., Maldonado, J., Maillard, J.P., Mall, U., Marquette, J.B., Mathis, S., Maxted, P., Matsuo, T., Medvedev, A., Miguel, Y., Minier, V., Morello, G., Mura, A., Narita, N., Nascimbeni, V., Nguyen Tong, N., Noce, V., Oliva, F., Palle, E., Palmer, P., Pancrazzi, M., Papageorgiou, A., Parmentier, V., Perger, M., Petralia, A., Pezzuto, S., Pierrehumbert, R., Pillitteri, I., Piotto, G., Pisano, G., Prisinzano, L., Radioti, A., Réess, J.M., Rezac, L., Rocchetto, M., Rosich, A., Sanna, N., Santerne, A., Savini, G., Scandariato, G., Sicardy, B., Sierra, C., Sindoni, G., Skup, K., Snellen, I., Sobiecki, M., Soret, L., Sozzetti, A., Stiepen, A., Strugarek, A., Taylor, J., Taylor, W., Terenzi, L., Tessenyi, M., Tsiaras, A., Tucker, C., Valencia, D., Vasisht, G., Vazan, A., Vilardell, F., Vinatier, S., Viti, S., Waters, R., Wawer, P., Wawrzaszek, A., Whitworth, A., Yung, Y.L., Yurchenko, S.N., Osorio, M.R.Z., Zellem, R., Zingales, T., Zwart, F.: A chemical survey of exoplanets with ARIEL. Exp. Astron. 46, 135 (2018). https://doi.org/10.1007/s10686-018-9598-X

13. Erard, S., Cecconi, B., Le Sidaner, P., Rossi, A.P., Capria, M.T., Schmitt, B., Génot, V., André, N., Vandaele, A.C., Scherf, M., Hueso, R., Määttänen, A., Thuillot, W., Carry, B., Achilleos, N., Marmo, C., Santolik, O., Benson, K., Fernique, P., Beigbeder, L., Millour, E., Rousseau, B., Andrieu, F., Chauvin, C., Minin, M., Ivanoski, S., Longobardo, A., Bollard, P., Albert, D., Gangloff, M., Jourdane, N., Bouchemit, M., Glorian, J.M., Trompet, L., Al-Ubaidi, T., Juaristi, J., Desmars, J., Guio, P., Delaa, O., Lagain, A., Soucek, J., Pisa, D.: VESPA: A community-driven virtual observatory in planetary science. Planet. Space Sci. 150, 65 (2018). https://doi.org/10.1016/j.pss.2017.05.013

14. Edwards, B., Mugnai, L., Tinetti, G., Pascale, E., Sarkar, S.: An updated study of potential targets for Ariel. AJ 157(6), 242 (2019). https://doi.org/10.3847/1538-3881/ab1cb9

15. Bonney, R., Cooper, C.B., Dickinson, J., Kelling, S., Phillips, T., Rosenberg, K.V., Shirk, J.: Citizen science: A developing tool for expanding science knowledge and scientific literacy. BioScience 59(11), 977 (2009). https://doi.org/10.1525/bio.2009.59.11.9

16. Kosmala, M., Wiggins, A., Swanson, A., Simmons, B.: Assessing data quality in citizen science. Front. Ecol. Environ. 14(10), 551 (2016). https://doi.org/10.1002/fee.1436

17. Aceves-Bueno, E., Adeleye, A.S., Feraud, M., Huang, Y., Tao, M., Yang, Y., Anderson, S.E.: The accuracy of citizen science data: A quantitative review. Bull. Ecol. Soc. Am. 98(4), 278 (2017). https://doi.org/10.1002/bes2.1336

18. Freitag, A., Meyer, R., Whiteman, L.: Strategies employed by citizen science programs to increase the credibility of their data. Citizen Sci. Theory Pract. 1(1), 2 (2016). https://doi.org/10.5334/cstp.6

19. Bouma, L.G., Winn, J.N., Baxter, C., Bhatti, W., Dai, F., Daylan, T., Désert, J.M., Hill, M.L., Kane, S.R., Stassun, K.G., Villasenor, J., Ricker, G.R., Vanderspek, R., Latham, D.W., Seager, S., Jenkins, J.M., Berta-Thompson, Z., Colón, K., Fausnaugh, M., Glidden, A., Guerrero, N., Rodriguez, J.E., Twicken, J.D., Wohler, B.: WASP-4b arrived early for the TESS mission. AJ 157(6), 217 (2019). https://doi.org/10.3847/1538-3881/ab189f 
20. Smith, A.M.S., Eigmüller, P., Oshagh, M.: A new orbital ephemeris for WASP-128b. J. Southworth Res. Notes Am. Astron. Soc. 4(2), 23 (2020). https://doi.org/10.3847/2515-5172/ab7343

21. Livingston, J.H., Crossfield, I.J.M., Werner, M.W., Gorjian, V., Petigura, E.A., Ciardi, D.R., Dressing, C.D., Fulton, B.J., Hirano, T., Schlieder, J.E.: Spitzer observations confirm and rescue the habitable-zone super-earth K2-18b for future characterization. AJ 157(3), 102 (2019). https://doi.org/10.3847/1538-3881/aaff69

22. Kosiarek, M.R., Crossfield, I.J.M., Hardegree-Ullman, K.K., Livingston, J.H., Benneke, B., Henry, G.W., Howard, W.S., Berardo, D., Blunt, S., Fulton, B.J.: Bright opportunities for atmospheric characterization of small planets: Masses and radii of K2-3 b, c, and d and GJ3470 b from radial velocity measurements and Spitzer transits. AJ 157(3), 97 (2019). https://doi.org/10.3847/1538-3881/aaf79c

23. Skaf, N., Fabienne Bieger, M., Edwards, B., Changeat, Q., Morvan, M., Kiefer, F., Blain, D., Zingales, T., Poveda, M., Al-Refaie, A., Baeyens, R., Gressier, A., Guilluy, G., Yassin Jaziri, A., Modirrousta-Galian, D., Mugnai, L.V., Pluriel, W., Whiteford, N., Wright, S., Hou Yip, K., Charnay, B., Leconte, J., Drossart, P., Tsiaras, A., Venot, O., Waldmann, I.: ARES II: Characterising the hot jupiters WASP-127 b, WASP-79 b and WASP-62 b with HST. J.P. Beaulieu, arXiv e-prints arXiv 2005, 09615 (2020)

24. Bruno, G., Lewis, N.K., Stevenson, K.B., Filippazzo, J., Hill, M., Fraine, J.D., Wakeford, H.R., Deming, D., Kilpatrick, B., Line, M.R., Morley, C.V., Collins, K.A., Conti, D.M., Garlitz, J., Rodriguez, J.E.: A comparative study of WASP-67 b and HAT-P-38 b from WFC3 data. AJ 155, 55 (2018). https://doi.org/10.3847/1538-3881/aaa0c7

25. Broeg, C., Fortier, A., Ehrenreich, D., Alibert, Y., Baumjohann, W., Benz, W., Deleuil, M., Gillon, M., Ivanov, A., Liseau, R., Meyer, M., Oloffson, G., Pagano, I., Piotto, G., Pollacco, D., Queloz, D., Ragazzoni, R., Renotte, E., Steller, M., Thomas, N.: CHEOPS: A transit photometry mission for ESA's small mission programme. In: European physical journal web of conferences, vol. 47, p. 03005 (2013). https://doi.org/10.1051/epjconf/20134703005

26. Poddaný, S., Brát, L., Pejcha, O.: Exoplanet transit database. Reduction and processing of the photometric data of exoplanet transits. New Astron. 15(3), 297 (2010). https://doi.org/10.1016/j.newast.2009.09.001

27. Tsiaras, A., Waldmann, I.P., Rocchetto, M., Varley, R., Morello, G., Damiano, M., Tinetti, G.: pylightcurve: Exoplanet lightcurve model (2016)

28. Tsiaras, A., Waldmann, I.P., Zingales, T., Rocchetto, M., Morello, G., Damiano, M., Karpouzas, K., Tinetti, G., McKemmish, L.K., Tennyson, J., Yurchenko, S.N.: A population study of gaseous exoplanets. AJ 155, 156 (2018). https://doi.org/10.3847/1538-3881/aaaf75

29. Morello, G., Claret, A., Martin-Lagarde, M., Cossou, C., Tsiaras, A., Lagage, P.O.: The ExoTETHyS package: tools for Exoplanetary transits around host stars. AJ 159(2), 75 (2020). https://doi.org/10.3847/1538-3881/ab63dc

30. Claret, A.: A new non-linear limb-darkening law for LTE stellar atmosphere models. Calculations for $-5.0<=\log [\mathrm{M} / \mathrm{H}]<=+1,2000 \mathrm{~K}<=\mathrm{T}_{e f f}<=50000 \mathrm{~K}$ at several surface gravities. Astron. Astrophys. 363, 1081 (2000)

31. Astropy Collaboration, Price-Whelan, A.M., Sipőcz, B.M., Günther, H.M., Lim, P.L., Crawford, S.M., Conseil, S., Shupe, D.L., Craig, M.W., Dencheva, N., Ginsburg, A., Vand erPlas, J.T., Bradley, L.D., Pérez-Suárez, D., de Val-Borro, M., Aldcroft, T.L., Cruz, K.L., Robitaille, T.P., Tollerud, E.J., Ardelean, C., Babej, T., Bach, Y.P., Bachetti, M., Bakanov, A.V., Bamford, S.P., Barentsen, G., Barmby, P., Baumbach, A., Berry, K.L., Biscani, F., Boquien, M., Bostroem, K.A., Bouma, L.G., Brammer, G.B., Bray, E.M., Breytenbach, H., Buddelmeijer, H., Burke, D.J., Calderone, G., Cano Rodríguez, J.L., Cara, M., Cardoso, J.V.M., Cheedella, S., Copin, Y., Corrales, L., Crichton, D., D’Avella, D., Deil, C., Depagne, É., Dietrich, J.P., Donath, A., Droettboom, M., Earl, N., Erben, T., Fabbro, S., Ferreira, L.A., Finethy, T., Fox, R.T., Garrison, L.H., Gibbons, S.L.J., Goldstein, D.A., Gommers, R., Greco, J.P., Greenfield, P., Groener, A.M., Grollier, F., Hagen, A., Hirst, P., Homeier, D., Horton, A.J., Hosseinzadeh, G., Hu, L., Hunkeler, J.S., Ivezić, Ž., Jain, A., Jenness, T., Kanarek, G., Kendrew, S., Kern, N.S., Kerzendorf, W.E., Khvalko, A., King, J., Kirkby, D., Kulkarni, A.M., Kumar, A., Lee, A., Lenz, D., Littlefair, S.P., Ma, Z., Macleod, D.M., Mastropietro, M., McCully, C., Montagnac, S., Morris, B.M., Mueller, M., Mumford, S.J., Muna, D., Murphy, N.A., Nelson, S., Nguyen, G.H., Ninan, J.P., Nöthe, M., Ogaz, S., Oh, S., Parejko, J.K., Parley, N., Pascual, S., Patil, R., Patil, A.A., Plunkett, A.L., Prochaska, J.X., Rastogi, T., Reddy Janga, V., Sabater, J., Sakurikar, P., Seifert, M., Sherbert, L.E., Sherwood-Taylor, H., Shih, A.Y., Sick, J., Silbiger, M.T., Singanamalla, S., Singer, L.P., Sladen, P.H., Sooley, K.A., Sornarajah, S., Streicher, O., Teuben, P., Thomas, 
S.W., Tremblay, G.R., Turner, J.E.H., Terrón, V., van Kerkwijk, M.H., de la Vega, A., Watkins, L.L., Weaver, B.A., Whitmore, J.B., Woillez, J., Zabalza, V., Astropy Contributors: The Astropy project: building an open-science project and status of the v2.0 core package. AJ 156(3), 123 (2018). https://doi.org/10.3847/1538-3881/aabc4f

32. Foreman-Mackey, D., Hogg, D.W., Lang, D., Goodman, J.: emcee: The MCMC hammer. Publ. Astron. Soc. Pac. 125(925), 306 (2013). https://doi.org/10.1086/670067

33. Hunter, J.D.: Matplotlib: A 2D graphics environment. Comput. Sci. Eng. 9(3), 90 (2007). https://doi.org/10.1109/MCSE.2007.55

34. Oliphant, T.E.: A guide to NumPy, vol. 1. Trelgol Publishing, USA (2006)

35. Virtanen, P., Gommers, R., Oliphant, T.E., Haberland, M., Reddy, T., Cournapeau, D., Burovski, E., Peterson, P., Weckesser, W., Bright, J., van der Walt, S.J., Brett, M., Wilson, J., Jarrod Millman, K., Mayorov, N., Nelson, A.R.J., Jones, E., Kern, R., Larson, E., Carey, C., Polat, İ., Feng, Y., Moore, E.W., Vand erPlas, J., Laxalde, D., Perktold, J., Cimrman, R., Henriksen, I., Quintero, E.A., Harris, C.R., Archibald, A.M., Ribeiro, A.H., Pedregosa, F., van Mulbregt, P., S... Contributors: SciPy 1.0: Fundamental algorithms for scientific computing in python. Nat. Methods 17, 261 (2020). https:// doi.org/10.1038/s41592-019-0686-2

36. Bakos, G.Á., Hartman, J., Torres, G., Latham, D.W., Kovács, G., Noyes, R.W., Fischer, D.A., Johnson, J.A., Marcy, G.W., Howard, A.W., Kipping, D., Esquerdo, G.A., Shporer, A., Béky, B., Buchhave, L.A., Perumpilly, G., Everett, M., Sasselov, D.D., Stefanik, R.P., Lázár, J., Papp, I., Sári, P.: HAT-P-20b-HAT-P-23b: Four massive transiting extrasolar planets. ApJ 742(2), 116 (2011). https://doi.org/10.1088/0004-637X/742/2/116

37. Sun, L., Gu, S., Wang, X., Collier Cameron, A., Cao, D., Wang, Y., Xiang, Y., Hui, H.K., Kwok, C.T., Yeung, B., Ng, E., Grau Horta, F.: Refined system parameters and TTV study of transiting exoplanetary system HAT-P-20. AJ 153(1), 28 (2017). https://doi.org/10.3847/1538-3881/153/1/28

38. Granata, V., Nascimbeni, V., Piotto, G., Bedin, L.R., Borsato, L., Cunial, A., Damasso, M., Malavolta, L.: TASTE IV: Refining ephemeris and orbital parameters for HAT-P-20b and WASP-1b. Astronomische Nachrichten 335(8), 797 (2014). https://doi.org/10.1002/asna.201412072

39. Esposito, M., Covino, E., Desidera, S., Mancini, L., Nascimbeni, V., Zanmar Sanchez, R., Biazzo, K., Lanza, A.F., Leto, G., Southworth, J., Bonomo, A.S., Suárez Mascareño, A., Boccato, C., Cosentino, R., Claudi, R.U., Gratton, R., Maggio, A., Micela, G., Molinari, E., Pagano, I., Piotto, G., Poretti, E., Smareglia, R., Sozzetti, A., Affer, L., Anderson, D.R., Andreuzzi, G., Benatti, S., Bignamini, A., Borsa, F., Borsato, L., Ciceri, S., Damasso, M., di Fabrizio, L., Giacobbe, P., Granata, V., Harutyunyan, A., Henning, T., Malavolta, L., Maldonado, J., Martinez Fiorenzano, A., Masiero, S., Molaro, P., Molinaro, M., Pedani, M., Rainer, M., Scandariato, G., Turner, O.D.: The GAPS Programme with HARPS-N at TNG. XIII. The orbital obliquity of three close-in massive planets hosted by dwarf K-type stars: WASP-43, HAT-P-20 and Qatar-2. Astron. Astrophys. 601, A53 (2017). https://doi.org/10.1051/0004-6361/201629720

40. Turner, J.D., Pearson, K.A., Biddle, L.I., Smart, B.M., Zellem, R.T., Teske, J.K., Hardegree-Ullman, K.K., Griffith, C.C., Leiter, R.M., Cates, I.T., Nieberding, M.N., Smith, C.T.W., Thompson, R.M., Hofmann, R., Berube, M.P., Nguyen, C.H., Small, L.C., Guvenen, B.C., Richardson, L., McGraw, A., Raphael, B., Crawford, B.E., Robertson, A.N., Tombleson, R., Carleton, T.M., Towner, A.P.M., Walker-LaFollette, A.M., Hume, J.R., Watson, Z.T., Jones, C.K., Lichtenberger, M.J., Hoglund, S.R., Cook, K.L., Crossen, C.A., Jorgensen, C.R., Romine, J.M., Thompson, A.R., Villegas, C.F., Wilson, A.A., Sanford, B., Taylor, J.M., Henz, T.N.: Ground-based near-UV observations of 15 transiting exoplanets: constraints on their atmospheres and no evidence for asymmetrical transits. MNRAS 459(1), 789 (2016). https://doi.org/10.1093/mnras/stw574

41. Kipping, D.M., Bakos, G.Á.., Hartman, J., Torres, G., Shporer, A., Latham, D.W., Kovács, G., Noyes, R.W., Howard, A.W., Fischer, D.A., Johnson, J.A., Marcy, G.W., Béky, B., Perumpilly, G., Esquerdo, G.A., Sasselov, D.D., Stefanik, R.P., Lázár, J., Papp, I., Sári, P.: HAT-P-24b: an inflated hot jupiter on a 3.36 day period transiting a hot, metal-poor star. ApJ 725(2), 2017 (2010). https://doi.org/10.1088/0004-637X/725/2/2017

42. Buchhave, L.A., Bakos, G.Á.., Hartman, J.D., Torres, G., Latham, D.W., Andersen, J., Kovács, G., Noyes, R.W., Shporer, A., Esquerdo, G.A., Fischer, D.A., Johnson, J.A., Marcy, G.W., Howard, A.W., Béky, B., Sasselov, D.D., Fürész, G., Quinn, S.N., Stefanik, R.P., Szklenár, T., Berlind, P., Calkins, M.L., Lázár, J., Papp, I., Sári, P.: Hat-P-28b and Hat-P-29b: Two sub-jupiter mass transiting planets. ApJ 733(2), 116 (2011). https://doi.org/10.1088/0004-637X/733/2/116 
43. Hartman, J.D., Bhatti, W., Bakos, G.Á., Bieryla, A., Kovács, G., Latham, D.W., Csubry, Z., de Val-Borro, M., Penev, K., Buchhave, L.A., Torres, G., Howard, A.W., Marcy, G.W., Johnson, J.A., Isaacson, H., Sato, B., Boisse, I., Falco, E., Everett, M.E., Szklenar, T., Fulton, B.J., Shporer, A., Kovács, T., Hansen, T., Béky, B., Noyes, R.W., Lázár, J., Papp, I., Sári, P.: HAT-P-50b, HAT-P-51b, HAT-P-52b, and HAT-P-53b: Three transiting hot jupiters and a transiting hot saturn from the hatnet survey. AJ 150(6), 168 (2015). https://doi.org/10.1088/0004-6256/150/6/168

44. Bakos, G.Á., Hartman, J.D., Bhatti, W., Bieryla, A., de Val-Borro, M., Latham, D.W., Buchhave, L.A., Csubry, Z., Penev, K., Kovács, G., Béky, B., Falco, E., Kovács, T., Howard, A.W., Johnson, J.A., Isaacson, H., Marcy, G.W., Torres, G., Noyes, R.W., Berlind, P., Calkins, M.L., Esquerdo, G.A., Lázár, J., Papp, I., Sári, P.: HAT-P-54b: A hot jupiter transiting a $0.6 \mathrm{M}_{s}$ star in field 0 of the K2 mission. AJ 149(4), 149 (2015). https://doi.org/10.1088/0004-6256/149/4/149

45. Johnson, M.C., Gandolfi, D., Fridlund, M., Csizmadia, S., Endl, M., Cabrera, J., Cochran, W.D., Deeg, H.J., Grziwa, S., Ramírez, I., Hatzes, A.P., Eigmüller, P., Barragán, O., Erikson, A., Guenther, E.W., Korth, J., Kuutma, T., Nespral, D., Pätzold, M., Palle, E., Prieto-Arranz, J., Rauer, H., Saario, J.: Two hot jupiters from K2 campaign 4. AJ 151(6), 171 (2016). https://doi.org/10.3847/0004-6256/151/6/171

46. Rodriguez, J.E., Colón, K.D., Stassun, K.G., Wright, D., Cargile, P.A., Bayliss, D., Pepper, J., Collins, K.A., Kuhn, R.B., Lund, M.B., Siverd, R.J., Zhou, G., Gaudi, B.S., Tinney, C.G., Penev, K., Tan, T.G., Stockdale, C., Curtis, I.A., James, D., Udry, S., Segransan, D., Bieryla, A., Latham, D.W., Beatty, T.G., Eastman, J.D., Myers, G., Bartz, J., Bento, J., Jensen, E.L.N., Oberst, T.E., Stevens, D.J.: KELT-14b and KELT-15b: An independent discovery of wasp-122b and a new hot jupiter. AJ1 151(6), 138 (2016). https://doi.org/10.3847/0004-6256/151/6/138

47. Edwards, B., Changeat, Q., Yip, K.H., Tsiaras, A., Taylor, J., Akhtar, B., AlDaghir, J., Bhattarai, P., Bhudia, T., Chapagai, A., Huang, M., Kabir, D., Khag, V., Khaliq, S., Khatri, K., Kneth, J., Kothari, M., Najmudin, I., Panchalingam, L., Patel, M., Premachand ran, L., Qayyum, A., Rana, P., Shaikh, Z., Syed, S., Theti, H., Zaidani, M., Saraf, M., de Mijolla, D., Caines, H., Kokori, A., Rocchetto, M., Mallonn, M., Bachschmidt, M., Bosch, J.M., Bretton, M., Chatelain, P., Deldem, M., Di Sisto, R., Evans, P., Fernández-Lajús, E., Guerra, P., Horta, F.G., Kang, W., Kim, T., Leroy, A., Lomoz, F., de Haro, J.L., Hentunen, V.P., Jongen, Y., Molina, D., Montaigut, R., Naves, R., Raetz, M., Sauer, T., Watkins, A., Wünsche, A., Zibar, M., Dunn, W., Tessenyi, M., Savini, G., Tinetti, G., Tennyson, A.: Original research by young twinkle students (ORBYTS): Ephemeris refinement of transiting exoplanets. MNRAS 4(7), 109 (2020). https://doi.org/10.1093/mnras/staa1245

48. Oberst, T.E., Rodriguez, J.E., Colón, K.D., Angerhausen, D., Bieryla, A., Ngo, H., Stevens, D.J., Stassun, K.G., Gaudi, B.S., Pepper, J., Penev, K., Mawet, D., Latham, D.W., Heintz, T.M., Osei, B.W., Collins, K.A., Kielkopf, J.F., Visgaitis, T., Reed, P.A., Escamilla, A., Yazdi, S., McLeod, K.K., Lunsford, L.T., Spencer, M., Joner, M.D., Gregorio, J., Gaillard, C., Matt, K., Dumont, M.T., Stephens, D.C., Cohen, D.H., Jensen, E.L.N., Calchi Novati, S., Bozza, V., Labadie-Bartz, J., Siverd, R.J., Lund, M.B., Beatty, T.G., Eastman, J.D., Penny, M.T., Manner, M., Zambelli, R., Fulton, B.J., Stockdale, C., DePoy, D.L., Marshall, J.L., Pogge, R.W., Gould, A., Trueblood, M., Trueblood, P.: KELT-16b: A highly irradiated, ultra-short period hot jupiter nearing tidal disruption. AJ 153(3), 97 (2017). https://doi.org/10.3847/1538-3881/153/3/97

49. Maciejewski, G., Fernández, M., Aceituno, F., Martín-Ruiz, S., Ohlert, J., Dimitrov, D., Szyszka, K., von Essen, C., Mugrauer, M., Bischoff, R., Michel, K.U., Mallonn, M., Stangret, M., Moździerski, D.: Planet-star interactions with precise transit timing. I. The refined orbital decay rate for wasp- $12 \mathrm{~b}$ and initial constraints for HAT-P-23 b, KELT-1 b, KELT-16 b, WASP-33 b and WASP-103 b. ACTA Astron. 68(4), 371 (2018). https://doi.org/10.32023/0001-5237/68.4.4

50. Patra, K.C., Winn, J.N., Holman, M.J., Gillon, M., Burdanov, A., Jehin, E., Delrez, L., Pozuelos, F.J., Barkaoui, K., Benkhaldoun, Z., Narita, N., Fukui, A., Kusakabe, N., Kawauchi, K., Terada, Y., Bouma, L.G., Weinberg, N.N., Broome, M.: The continuing search for evidence of tidal orbital decay of hot jupiters. AJ 159(4), 150 (2020). https://doi.org/10.3847/1538-3881/ab7374

51. Burdanov, A., Benni, P., Sokov, E., Krushinsky, V., Popov, A., Delrez, L., Gillon, M., Hébrard, G., Deleuil, M., Wilson, P.A., Demangeon, O., Baștürk, Ö., Pakštiene, E., Sokova, I., Rusov, S.A., Dyachenko, V.V., Rastegaev, D.A., Beskakotov, A., Marchini, A., Bretton, M., Shadick, S., Ivanov, K.: KPS-1b: The first transiting exoplanet discovered using an amateur astronomer's wide-field CCD data. Publ. Astron. Soc. Pac. 130(989), 074401 (2018). https://doi.org/10.1088/1538-3873/aabde2

52. Alsubai, K., Mislis, D., Tsvetanov, Z.I., Latham, D.W., Bieryla, A., Buchhave, L.A., Esquerdo, G.A., Bramich, D.M., Pyrzas, S., Vilchez, N.P.E., Mancini, L., Southworth, J., Evans, D.F., Henning, 
T., Ciceri, S.: Qatar exoplanet survey : Qatar-3b, Qatar-4b, and Qatar-5b. AJ 153(4), 200 (2017). https://doi.org/10.3847/1538-3881/aa6340

53. Skillen, I., Pollacco, D., Collier Cameron, A., Hebb, L., Simpson, E., Bouchy, F., Christian, D.J., Gibson, N.P., Hébrard, G., Joshi, Y.C., Loeillet, B., Smalley, B., Stempels, H.C., Street, R.A., Udry, S., West, R.G., Anderson, D.R., Barros, S.C.C., Enoch, B., Haswell, C.A., Hellier, C., Horne, K., Irwin, J., Keenan, F.P., Lister, T.A., Maxted, P., Mayor, M., Moutou, C., Norton, A.J., Parley, N., Queloz, D., Ryans, R., Todd, I., Wheatley, P.J., Wilson, D.M.: The $0.5 \mathrm{M}_{J}$ transiting exoplanet WASP-13b. Astron. Astrophys. 502(1), 391 (2009). https://doi.org/10.1051/0004-6361/200912018

54. Barros, S.C.C., Pollacco, D.L., Gibson, N.P., Keenan, F.P., Skillen, I., Steele, I.A.: High-precision transit observations of the exoplanet WASP-13b with the RISE instrument. MNRAS 419(2), 1248 (2012). https://doi.org/10.1111/j.1365-2966.2011.19784.x

55. Anderson, D.R., Collier Cameron, A., Hellier, C., Lendl, M., Lister, T.A., Maxted, P.F.L., Queloz, D., Smalley, B., Smith, A.M.S., Triaud, A.H.M.J., West, R.G., Brown, D.J.A., Gillon, M., Pepe, F., Pollacco, D., Ségransan, D., Street, R.A., Udry, S.: WASP-31b: a low-density planet transiting a metal-poor, late-F-type dwarf star. Astron. Astrophys. 531, A60 (2011). https://doi.org/10.1051/0004-6361/201016208

56. Dragomir, D., Kane, S.R., Pilyavsky, G., Mahadevan, S., Ciardi, D.R., Gazak, J.Z., Gelino, D.M., Payne, A., Rabus, M., Ramirez, S.V., von Braun, K., Wright, J.T., Wyatt, P.: TERMS photometry of known transiting exoplanets. AJ 142(4), 115 (2011). https://doi.org/10.1088/0004-6256/142/4/115

57. Sing, D.K., Wakeford, H.R., Showman, A.P., Nikolov, N., Fortney, J.J., Burrows, A.S., Ballester, G.E., Deming, D., Aigrain, S., Désert, J.M., Gibson, N.P., Henry, G.W., Knutson, H., Lecavelier des Etangs, A., Pont, F., Vidal-Madjar, A., Williamson, M.W., Wilson, P.A.: HST hot-Jupiter transmission spectral survey: detection of potassium in WASP-31b along with a cloud deck and Rayleigh scattering. MNRAS 446(3), 2428 (2015). https://doi.org/10.1093/mnras/stu2279

58. Hellier, C., Anderson, D.R., Collier Cameron, A., Doyle, A.P., Fumel, A., Gillon, M., Jehin, E., Lendl, M., Maxted, P.F.L., Pepe, F., Pollacco, D., Queloz, D., Ségransan, D., Smalley, B., Smith, A.M.S., Southworth, J., Triaud, A.H.M.J., Udry, S., West, R.G.: Seven transiting hot Jupiters from WASP-South, Euler and TRAPPIST: WASP-47b, WASP-55b, WASP-61b, WASP-62b, WASP-63b, WASP-66b and WASP-67b. MNRAS 426(1), 739 (2012). https://doi.org/10.1111/j.1365-2966.2012.21780.x

59. Mancini, L., Southworth, J., Ciceri, S., Calchi Novati, S., Dominik, M., Henning, T., Jørgensen, U.G., Korhonen, H., Nikolov, N., Alsubai, K.A., Bozza, V., Bramich, D.M., D’Ago, G., Figuera Jaimes, R., Galianni, P., Gu, S.H., Harps $\varnothing$, e., K., Hinse, T.C., Hundertmark, M., Juncher, D., Kains, N., Popovas, A., Rabus, M., Rahvar, S., Skottfelt, J., Snodgrass, C., Street, R., Surdej, J., Tsapras, Y., Vilela, C., Wang, X.B., Wertz, O.: Physical properties of the WASP-67 planetary system from multi-colour photometry. Astron. Astrophys. 568, A127 (2014). https://doi.org/10.1051/0004-6361/201424106

60. Bruno, G., Lewis, N.K., Stevenson, K.B., Filippazzo, J., Hill, M., Fraine, J.D., Wakeford, H.R., Deming, D., Kilpatrick, B., Line, M.R., Morley, C.V., Collins, K.A., Conti, D.M., Garlitz, J., Rodriguez, J.E.: A Comparative Study of WASP-67 b and HAT-P-38 b from WFC3 Data. AJ 155(2), 55 (2018). https://doi.org/10.3847/1538-3881/aaa0c7

61. Gómez Maqueo Chew, Y., Faedi, F., Pollacco, D., Brown, D.J.A., Doyle, A.P., Collier Cameron, A., Gillon, M., Lendl, M., Smalley, B., Triaud, A.H.M.J., West, R.G., Wheatley, P.J., Busuttil, R., Liebig, C., Anderson, D.R., Armstrong, D.J., Barros, S.C.C., Bento, J., Bochinski, J., Burwitz, V., Delrez, L., Enoch, B., Fumel, A., Haswell, C.A., Hébrard, G., Hellier, C., Holmes, S., Jehin, E., Kolb, U., Maxted, P.F.L., McCormac, J., Miller, G.R.M., Norton, A.J., Pepe, F., Queloz, D., Rodríguez, J., Ségransan, D., Skillen, I., Stassun, K.G., Udry, S., Watson, C.: Discovery of WASP-65b and WASP-75b: Two hot Jupiters without highly inflated radii. Astron. Astrophys. 559, A36 (2013). https://doi.org/10.1051/0004-6361/201322314

62. Clark, B.J.M., Anderson, D.R., Hellier, C., Turner, O.D., Močnik, T.: An Analysis of Transiting Hot Jupiters Observed with K2: WASP-55b and WASP-75b. Publ. Astron. Soc. Pac. 130(985), 034401 (2018). https://doi.org/10.1088/1538-3873/aaa33e

63. Maxted, P.F.L., Anderson, D.R., Collier Cameron, A., Doyle, A.P., Fumel, A., Gillon, M., Hellier, C., Jehin, E., Lendl, M., Pepe, F., Pollacco, D.L., Queloz, D., Ségransan, D., Smalley, B., Southworth, K., Smith, A.M.S., Triaud, A.H.M.J., Udry, S., West, R.G.: WASP-77 Ab: A transiting hot jupiter planet in a wide binary system. Publ. Astron. Soc. Pac. 125(923), 48 (2013). https://doi.org/10.1086/669231 
64. Hellier, C., Anderson, D.R., Collier Cameron, A., Delrez, L., Gillon, M., Jehin, E., Lendl, M., Maxted, P.F.L., Pepe, F., Pollacco, D., Queloz, D., Ségransan, D., Smalley, B., Smith, A.M.S., Southworth, J., Triaud, A.H.M.J., Turner, O.D., Udry, S., West, R.G.: Three WASPsouth transiting exoplanets: WASP-74b, WASP-83b, and WASP-89b. AJ 150(1), 18 (2015). https://doi.org/10.1088/0004-6256/150/1/18

65. Smith, A.M.S., Anderson, D.R., Armstrong, D.J., Barros, S.C.C., Bonomo, A.S., Bouchy, F., Brown, D.J.A., Collier Cameron, A., Delrez, L., Faedi, F., Gillon, M., Gómez Maqueo Chew, Y., Hébrard, G., Jehin, E., Lendl, M., Louden, T.M., Maxted, P.F.L., Montagnier, G., Neveu-VanMalle, M., Osborn, H.P., Pepe, F., Pollacco, D., Queloz, D., Rostron, J.W., Segransan, D., Smalley, B., Triaud, A.H.M.J., Turner, O.D., Udry, S., Walker, S.R., West, R.G., Wheatley, P.J.: WASP-104b and WASP-106b: two transiting hot Jupiters in 1.75-day and 9.3-day orbits. Astron. Astrophys. 570, A64 (2014). https://doi.org/10.1051/0004-6361/201424752

66. Močnik, T., Hellier, C., Southworth, J.: WASP-104b is darker than charcoal. AJ 156(2), 44 (2018). https://doi.org/10.3847/1538-3881/aacb26

67. Crouzet, N., McCullough, P.R., Long, D., Montanes Rodriguez, P., Lecavelier des Etangs, A., Ribas, I., Bourrier, V., Hébrard, G., Vilardell, F., Deleuil, M., Herrero, E., Garcia-Melendo, E., Akhenak, L., Foote, J., Gary, B., Benni, P., Guillot, T., Conjat, M., Mékarnia, D., Garlitz, J., Burke, C.J., Courcol, B., Demangeon, O.: Discovery of XO-6b: A hot jupiter transiting a fast rotating $\mathrm{f} 5$ star on an oblique orbit. AJ 153(3), 94 (2017). https://doi.org/10.3847/1538-3881/153/3/94

68. Johnson, J.A., Winn, J.N., Bakos, G.Á. ., Hartman, J.D., Morton, T.D., Torres, G., Kovács, G., Latham, D.W., Noyes, R.W., Sato, B., Esquerdo, G.A., Fischer, D.A., Marcy, G.W., Howard, A.W., Buchhave, L.A., Fưrész, G., Quinn, S.N., Béky, B., Sasselov, D.D., Stefanik, R.P., Lázár, J., Papp, I., Sári, P.: HAT-P-30b: A Transiting Hot Jupiter on a Highly Oblique Orbit. ApJ 735(1), 24 (2011). https://doi.org/10.1088/0004-637X/735/1/24

69. Enoch, B., Anderson, D.R., Barros, S.C.C., Brown, D.J.A., Collier Cameron, A., Faedi, F., Gillon, M., Hébrard, G., Lister, T.A., Queloz, D., Santerne, A., Smalley, B., Street, R.A., Triaud, A.H.M.J., West, R.G., Bouchy, F., Bento, J., Butters, O., Fossati, L., Haswell, C.A., Hellier, C., Holmes, S., Jehin, E., Lendl, M., Maxted, P.F.L., McCormac, J., Miller, G.R.M., Moulds, V., Moutou, C., Norton, A.J., Parley, N., Pepe, F., Pollacco, D., Segransan, D., Simpson, E., Skillen, I., Smith, A.M.S., Udry, S., Wheatley, P.J.: WASP-35b, WASP-48b, and HAT-P-30b/WASP51b: Two new planets and an independent discovery of a hat planet. AJ 142(3), 86 (2011). https://doi.org/10.1088/0004-6256/142/3/86

70. Maciejewski, G., Dimitrov, D., Mancini, L., Southworth, J., Ciceri, S., D’Ago, G., Bruni, I., Raetz, S., Nowak, G., Ohlert, J., Puchalski, D., Saral, G., Derman, E., Petrucci, R., Jofre, E., Seeliger, M., Henning, T.: New transit observations for HAT-P-30 b, HAT-P-37 b, TrES-5 b, WASP-28 b, WASP-36 b and WASP-39 b. ACTA Astron. 66(1), 55 (2016)

71. Bakos, G.Á. ., Hartman, J.D., Torres, G., Béky, B., Latham, D.W., Buchhave, L.A., Csubry, Z., Kovács, G., Bieryla, A., Quinn, S., Szklenár, T., Esquerdo, G.A., Shporer, A., Noyes, R.W., Fischer, D.A., Johnson, J.A., Howard, A.W., Marcy, G.W., Sato, B., Penev, K., Everett, M., Sasselov, D.D., Fưrész, G., Stefanik, R.P., Lázár, J., Papp, I., Sári, P.: HAT-P-34b-HAT-P-37b: Four transiting planets more massive than jupiter orbiting moderately bright stars. AJ 144(1), 19 (2012). https://doi.org/10.1088/0004-6256/144/1/19

72. Turner, J.D., Leiter, R.M., Biddle, L.I., Pearson, K.A., Hardegree-Ullman, K.K., Thompson, R.M., Teske, J.K., Cates, I.T., Cook, K.L., Berube, M.P., Nieberding, M.N., Jones, C.K., Raphael, B., Wallace, S., Watson, Z.T., Johnson, R.E.: Investigating the physical properties of transiting hot Jupiters with the 1.5-m Kuiper Telescope. MNRAS 472(4), 3871 (2017). https://doi.org/10.1093/mnras/stx2221

73. Huang, C.X., Hartman, J.D., Bakos, G.Á.., Penev, K., Bhatti, W., Bieryla, A., de Val-Borro, M., Latham, D.W., Buchhave, L.A., Csubry, Z., Kovács, G., Béky, B., Falco, E., Berlind, P., Calkins, M.L., Esquerdo, G.A., Lázár, J., Papp, I., Sári, P.: HAT-P-56b: An inflated massive hot jupiter transiting a bright $\mathrm{F}$ star followed Up with K2 campaign 0 observations. AJ 150(3), 85 (2015). https://doi.org/10.1088/0004-6256/150/3/85

74. Bieryla, A., Collins, K., Beatty, T.G., Eastman, J., Siverd, R.J., Pepper, J., Gaudi, B.S., Stassun, K.G., Cañas, C., Latham, D.W., Buchhave, L.A., Sanchis-Ojeda, R., Winn, J.N., Jensen, E.L.N., Kielkopf, J.F., McLeod, K.K., Gregorio, J., Colón, K.D., Street, R., Ross, R., Penny, M., Mellon, S.N., Oberst, T.E., Fulton, B.J., Wang, J., Berlind, P., Calkins, M.L., Esquerdo, G.A., DePoy, D.L., Gould, A., 
Marshall, J., Pogge, R., Trueblood, M., Trueblood, P.: KELT-7b: A hot jupiter transiting a bright V = 8.54 rapidly rotating F-star. AJ 150(1), 12 (2015). https://doi.org/10.1088/0004-6256/150/1/12

75. Pollacco, D., Skillen, I., Collier Cameron, A., Loeillet, B., Stempels, H.C., Bouchy, F., Gibson, N.P., Hebb, L., Hébrard, G., Joshi, Y.C., McDonald, I., Smalley, B., Smith, A.M.S., Street, R.A., Udry, S., West, R.G., Wilson, D.M., Wheatley, P.J., Aigrain, S., Alsubai, K., Benn, C.R., Bruce, V.A., Christian, D.J., Clarkson, W.I., Enoch, B., Evans, A., Fitzsimmons, A., Haswell, C.A., Hellier, C., Hickey, S., Hodgkin, S.T., Horne, K., Hrudková, M., Irwin, J., Kane, S.R., Keenan, F.P., Lister, T.A., Maxted, P., Mayor, M., Moutou, C., Norton, A.J., Osborne, J.P., Parley, N., Pont, F., Queloz, D., Ryans, R., Simpson, E.: WASP-3b: a strongly irradiated transiting gas-giant planet. MNRAS 385(3), 1576 (2008). https://doi.org/10.1111/j.1365-2966.2008.12939.x

76. Tripathi, A., Winn, J.N., Johnson, J.A., Howard, A.W., Halverson, S., Marcy, G.W., Holman, M.J., de Kleer, K.R., Carter, J.A., Esquerdo, G.A., Everett, M.E., Cabrera, N.E.: A prograde, low-inclination orbit for the very hot jupiter WASP-3b. ApJ 715(1), 421 (2010). https://doi.org/10.1088/0004-637X/715/1/421

77. Gibson, N.P., Pollacco, D., Simpson, E.K., Joshi, Y.C., Todd, I., Benn, C., Christian, D., Hrudková, M., Keenan, F.P., Meaburn, J., Skillen, I., Steele, I.A.: Updated parameters for the transiting exoplanet WASP-3b using RISE, a new fast camera for the liverpool telescope. Astron. Astrophys. 492(2), 603 (2008). https://doi.org/10.1051/0004-6361:200811015

78. Christiansen, J.L., Ballard, S., Charbonneau, D., Deming, D., Holman, M.J., Madhusudhan, N., Seager, S., Wellnitz, D.D., Barry, R.K., Livengood, T.A., Hewagama, T., Hampton, D.L., Lisse, C.M., A'Hearn, M.F.: System parameters, transit times, and secondary eclipse constraints of the exoplanet systems HAT-P-4, TrES-2, TrES-3, and WASP-3 from the NASA EPOXI mission of opportunity. ApJ 726(2), 94 (2011). https://doi.org/10.1088/0004-637X/726/2/94

79. Nascimbeni, V., Cunial, A., Murabito, S., Sada, P.V., Aparicio, A., Piotto, G., Bedin, L.R., Milone, A.P., Rosenberg, A., Zurlo, A., Borsato, L., Damasso, M., Granata, V., Malavolta, L.: TASTE. III. A homogeneous study of transit time variations in WASP-3b. Astron. Astrophys. 549, A30 (2013). https://doi.org/10.1051/0004-6361/201219601

80. Sada, P.V., Deming, D., Jennings, D.E., Jackson, B.K., Hamilton, C.M., Fraine, J., Peterson, S.W., Haase, F., Bays, K., Lunsford, A., O’Gorman, E.: Extrasolar planet transits observed at Kitt peak national observatory. Publ. Astron. Soc. Pac. 124(913), 212 (2012). https://doi.org/10.1086/665043

81. Montalto, M., Gregorio, J., Boué, G., Mortier, A., Boisse, I., Oshagh, M., Maturi, M., Figueira, P., Sousa, S., Santos, N.C.: A new analysis of the WASP-3 system: no evidence for an additional companion. MNRAS 427(4), 2757 (2012). https://doi.org/10.1111/j.1365-2966.2012.21926.x

82. Maciejewski, G., Dimitrov, D., Neuhäuser, R., Niedzielski, A., Raetz, S., Ginski, C., Adam, C., Marka, C., Moualla, M., Mugrauer, M.: MNRAS407(4), 2625 (2010). https://doi.org/10.1111/ j.1365-2966.2010.17099.x

83. Eibe, M.T., Cuesta, L., Ullán, A., Pérez-Verde, A., Navas, J.: MNRAS423(2), 1381 (2012). https://doi.org/10.1111/j.1365-2966.2012.20962.x

84. Maciejewski, G., Niedzielski, A., Wolszczan, A., Nowak, G., Neuhäuser, R., Winn, J.N., Deka, B., Adamów, M., Górecka, M., Fernández, M., Aceituno, F.J., Ohlert, J., Errmann, R., Seeliger, M., Dimitrov, D., Latham, D.W., Esquerdo, G.A., McKnight, L., Holman, M.J., Jensen, E.L.N., Kramm, U., Pribulla, T., Raetz, S., Schmidt, T.O.B., Gin- ski, C., Mottola, S., Hellmich, S., Adam, C., Gilbert, H., Mugrauer, M., Saral, G., Popov, V., Raetz, M.: AJ146(6), 147 (2013). https://doi.org/10.1088/ 0004-6256/146/6/147

85. Maciejewski, G., Stangret, M., Ohlert, J., Basaran, C.S., Maciejczak, J., Puciata-Mroczynska, M., Boulanger, E.: Information Bulletin on Variable Stars 6243, 1 (2018). https://doi.org/10.22444/ IBVS.6243

86. Lister, T.A., Anderson, D.R., Gillon, M., Hebb, L., Smalley, B.S., Triaud, A.H.M.J., Collier Cameron, A., Wilson, D.M., West, R.G., Bentley, S.J., Christian, D.J., Enoch, R., Haswell, C.A., Hellier, C., Horne, K., Irwin, J., Joshi, Y.C., Kane, S.R., Mayor, M., Maxted, P.F.L., Norton, A.J., Parley, N., Pepe, F., Pollacco, D., Queloz, D., Ryans, R., Segransan, D., Skillen, I., Street, R.A., Todd, I., Udry, S., Wheatley, P.J.: WASP-16b: A new jupiter-like planet transiting a southern solar analog. ApJ 703(1), 752 (2009). https://doi.org/10.1088/0004-637X/703/1/752

87. Southworth, J., Mancini, L., Browne, P., Burgdorf, M., Calchi Novati, S., Dominik, M., Gerner, T., Hinse, T.C., Jørgensen, U.G., Kains, N., Ricci, D., Schäfer, S., Schönebeck, F., Tregloan-Reed, J., Alsubai, K.A., Bozza, V., Chen, G., Dodds, P., Dreizler, S., Fang, X.S., Finet, F., Gu, S.H., Hardis, S., Harpsøe, K., Henning, T., Hundertmark, M., Jessen-Hansen, J., Kerins, E., Kjeldsen, H., 
Liebig, C., Lund, M.N., Lundkvist, M., Mathiasen, M., Nikolov, N., Penny, M.T., Proft, S., Rahvar, S., Sahu, K., Scarpetta, G., Skottfelt, J., Snodgrass, C., Surdej, J., Wertz, O.: High-precision photometry by telescope defocusing - V. WASP-15 and WASP-16. MNRAS 434(2), 1300 (2013). https://doi.org/10.1093/mnras/stt1089

88. Maxted, P.F.L., Anderson, D.R., Collier Cameron, A., Gillon, M., Hellier, C., Queloz, D., Smalley, B., Triaud, A.H.M.J., West, R.G., Enoch, R., Lister, T.A., Pepe, F., Pollacco, D.L., Ségransan, D., Skillen, I., Udry, S.: WASP-32b: A transiting hot jupiter planet orbiting a lithium-poor, solar-type star. Publ. Astron. Soc. Pac. 122(898), 1465 (2010). https://doi.org/10.1086/657658

89. Sun, L.L., Gu, S.H., Wang, X.B., Collier Cameron, A., Cao, D.T., Wang, Y.B., Xiang, Y., Hui, H.K., Kwok, C.T., Yeung, B., Leung, K.C.: Long-term transit timing monitoring and homogenous study of WASP-32. Res. Astron. Astrophys. 15(1), 117-126 (2015). https://doi.org/10.1088/1674-4527/15/1/011

90. Johns-Krull, C.M., McCullough, P.R., Burke, C.J., Valenti, J.A., Janes, K.A., Heasley, J.N., Prato, L., Bissinger, R., Fleenor, M., Foote, C.N., Garcia-Melendo, E., Gary, B.L., Howell, P.J., Mallia, F., Masi, G., Vanmunster, T.: XO-3b: A massive planet in an eccentric orbit transiting an F5 V star. ApJ 677(1), 657 (2008). https://doi.org/10.1086/528950

91. Winn, J.N., Holman, M.J., Torres, G., McCullough, P., Johns-Krull, C., Latham, D.W., Shporer, A., Mazeh, T., Garcia-Melendo, E., Foote, C., Esquerdo, G., Everett, M.: The transit light curve project. IX. Evidence for a smaller radius of the exoplanet XO-3b. ApJ 683(2), 1076 (2008). https://doi.org/10.1086/589737

92. Garai, Z., Pribulla, T., Hambálek, Ł., Kundra, E., Vaňko, M., Raetz, S., Seeliger, M., Marka, C., Gilbert, H.: Affordable echelle spectroscopy of the eccentric HAT-P-2, WASP-14, and XO3 planetary systems with a sub-meter-class telescope. Astronomische Nachr. 338(1), 35 (2017). https://doi.org/10.1002/asna.201613208

93. Hébrard, G., Bouchy, F., Pont, F., Loeillet, B., Rabus, M., Bonfils, X., Moutou, C., Boisse, I., Delfosse, X., Desort, M., Eggenberger, A., Ehrenreich, D., Forveille, T., Lagrange, A.M., Lovis, C., Mayor, M., Pepe, F., Perrier, C., Queloz, D., Santos, N.C., Ségransan, D., Udry, S., Vidal-Madjar, A.: Misaligned spin-orbit in the XO-3 planetary system? Astron. Astrophys. 488(2), 763 (2008). https://doi.org/10.1051/0004-6361:200810056

94. Winn, J.N., Johnson, J.A., Fabrycky, D., Howard, A.W., Marcy, G.W., Narita, N., Crossfield, I.J., Suto, Y., Turner, E.L., Esquerdo, G., Holman, M.J.: On the spin-orbit misalignment of the XO-3 exoplanetary system. ApJ 700(1), 302 (2009). https://doi.org/10.1088/0004-637X/700/1/302

95. McCullough, P.R., Burke, C.J., Valenti, J.A., Long, D., Johns-Krull, C.M., Machalek, P., Janes, K.A., Taylor, B., Gregorio, J., Foote, C.N., Gary, B.L., Fleenor, M., García-Melendo, E., Vanmunster, T.: XO-4b: An extrasolar planet transiting an f5v star. arXiv:0805.2921 (2008)

96. Narita, N., Hirano, T., Sanchis-Ojeda, R., Winn, J.N., Holman, M.J., Sato, B., Aoki, W., Tamura, M.: The Rossiter-McLaughlin effect of the transiting exoplanet XO-4b*. Publ. Astron. Soc. Jpn. 62(6), L61 (2010). https://doi.org/10.1093/pasj/62.6.L61

97. Villanueva, J., S., Eastman, J.D., Gaudi, B.S.: The dedicated monitor of exotransits (DEMONEX): Seven transits of XO-4b. ApJ 820(2), 87 (2016). https://doi.org/10.3847/0004-637X/820/2/87

98. Todorov, K.O., Deming, D., Knutson, H.A., Burrows, A., Sada, P.V., Cowan, N.B., Agol, E., Desert, J.M., Fortney, J.J., Charbonneau, D., Laughlin, G., Langton, J., Showman, A.P., Lewis, N.K.: Warm spitzer observations of three hot exoplanets: XO-4b, HAT-P-6b, and HAT-P-8b. ApJ 746(1), 111 (2012). https://doi.org/10.1088/0004-637X/746/1/111

99. Bruno, G., Deleuil, M., Almenara, J.M., Barros, S.C.C., Lanza, A.F., Montalto, M., Boisse, I., Santerne, A., Lagrange, A.M.: Disentangling planetary and stellar activity features in the CoRoT-2 light curve. N. Meunier, Astron. Astrophys. 595, A89 (2016). https://doi.org/10.1051/0004-6361/201527699

100. Cáceres, C., Kabath, P., Hoyer, S., Ivanov, V.D., Rojo, P., Girard, J.H., Miller-Ricci Kempton, E., Fortney, J.J., Minniti, D.: Ground-based transit observations of the super-Earth GJ 1214 b. Astron. Astrophys. 565, A7 (2014). https://doi.org/10.1051/0004-6361/201321087

101. Lanotte, A.A., Gillon, M., Demory, B.O., Fortney, J.J., Astudillo, N., Bonfils, X., Magain, P., Delfosse, X., Forveille, T., Lovis, C., Mayor, M., Neves, V., Pepe, F., Queloz, D., Santos, N., Udry, S.: A global analysis of Spitzer and new HARPS data confirms the loneliness and metal-richness of GJ 436 b. Astron. Astrophys. 572, A73 (2014). https://doi.org/10.1051/0004-6361/201424373

102. Nikolov, N., Sing, D.K., Pont, F., Burrows, A.S., Fortney, J.J., Ballester, G.E., Evans, T.M., Huitson, C.M., Wakeford, H.R., Wilson, P.A., Aigrain, S., Deming, D., Gibson, N.P., Henry, G.W., Knutson, 
H., Lecavelier des Etangs, A., Showman, A.P., Vidal-Madjar, A., Zahnle, K.: Hubble Space Telescope hot Jupiter transmission spectral survey: a detection of $\mathrm{Na}$ and strong optical absorption in HAT-P-1b. MNRAS 437(1), 46 (2014). https://doi.org/10.1093/mnras/stt1859

103. Chan, T., Ingemyr, M., Winn, J.N., Holman, M.J., Sanchis-Ojeda, R., Esquerdo, G., Everett, M.: The Transit Light-curve Project. XIV. Confirmation of Anomalous Radii for the Exoplanets TrES-4b, HAT-P-3b, and WASP-12b. AJ 141(6), 179 (2011). https://doi.org/10.1088/0004-6256/141/6/179

104. Southworth, J., Mancini, L., Maxted, P.F.L., Bruni, I., Tregloan-Reed, J., Barbieri, M., Ruocco, N., Wheatley, P.J.: Physical properties and radius variations in the HAT-P-5 planetary system from simultaneous four-colour photometry. MNRAS 422(4), 3099 (2012). https://doi.org/10.1111/j.1365-2966.2012.20828.x

105. Wang, Y.H., Wang, S., Hinse, T.C., Wu, Z.Y., Davis, A.B., Hori, Y., Yoon, J.N., Han, W., Nie, J.D., Liu, H.G., Zhang, H., Zhou, J.L., Wittenmyer, R.A., Peng, X.Y., Laughlin, G.: Transiting Exoplanet Monitoring Project (TEMP). V. Transit Follow Up for HAT-P-9b, HAT-P-32b, and HAT-P-36b. AJ 157(2), 82 (2019). https://doi.org/10.3847/1538-3881/aaf6b6

106. Sada, P.V., Ramón-Fox, F.G.: Exoplanet transits registered at the Universidad de Monterrey Observatory. I. HAT-P-12b, HAT-P-13b, HAT-P-16b, HAT-P-23b, and WASP-10b. Publ. Astron. Soc. Pac. 128(960), 024402 (2016). https://doi.org/10.1088/1538-3873/128/960/024402

107. Seeliger, M., Kitze, M., Errmann, R., Richter, S., Ohlert, J.M., Chen, W.P., Guo, J.K., Göğüs, E., Güver, T., Aydin, B., Mottola, S., Hellmich, S., Fernand ez, M., Aceituno, F.J., Dimitrov, D., Kjurkchieva, D., Jensen, E., Cohen, D., Kundra, E., Pribulla, T., Vaňko, M., Budaj, J., Mallonn, M., Wu, Z.Y., Zhou, X., Raetz, S., Adam, C., Schmidt, T.O.B., Ide, A., Mugrauer, M., Marschall, L., Hackstein, M., Chini, R., Haas, M., Ak, T., Güzel, E., Özdönmez, A., Ginski, C., Marka, C., Schmidt, J.G., Dincel, B., Werner, K., Dathe, A., Greif, J., Wolf, V., Buder, S., Pannicke, A., Puchalski, D., Neuhäuser, R.: Ground-based transit observations of the HAT-P-18, HAT-P-19, HAT-P-27/WASP40 and WASP-21 systems. MNRAS 451(4), 4060 (2015). https://doi.org/10.1093/mnras/stv1187

108. Hartman, J.D., Bakos, G.Á. ., Torres, G., Latham, D.W., Kovács, G., Béky, B., Quinn, S.N., Mazeh, T., Shporer, A., Marcy, G.W., Howard, A.W., Fischer, D.A., Johnson, J.A., Esquerdo, G.A., Noyes, R.W., Sasselov, D.D., Stefanik, R.P., Fernandez, J.M., Szklenár, T., Lázár, J., Papp, I., Sári, P.: HATP-32b and HAT-P-33b: Two highly inflated hot Jupiters transiting high-jitter stars. ApJ 742(1), 59 (2011). https://doi.org/10.1088/0004-637X/742/1/59

109. Mancini, L., Esposito, M., Covino, E., Raia, G., Southworth, J., Tregloan-Reed, J., Biazzo, K., Bonomo, A.S., Desidera, S., Lanza, A.F., Maciejewski, G., Poretti, E., Sozzetti, A., Borsa, F., Bruni, I., Ciceri, S., Claudi, R., Cosentino, R., Gratton, R., Martinez Fiorenzano, A.F., Lodato, G., Lorenzi, V., Marzari, F., Murabito, S., Affer, L., Bignamini, A., Bedin, L.R., Boccato, C., Damasso, M., Henning, T., Maggio, A., Micela, G., Molinari, E., Pagano, I., Piotto, G., Rainer, M., Scandariato, G., Smareglia, R., Zanmar Sanchez, R.: The GAPS Programme with HARPS-N at TNG. VIII. Observations of the Rossiter-McLaughlin effect and characterisation of the transiting planetary systems HAT-P-36 and WASP-11/HAT-P-10. Astron. Astrophys. 579, A136 (2015). https://doi.org/10.1051/0004-6361/201526030

110. Agol, E., Cowan, N.B., Knutson, H.A., Deming, D., Steffen, J.H., Henry, G.W., Charbonneau, D.: The climate of HD 189733b from fourteen transits and eclipses measured by Spitzer. ApJ 721(2), 1861 (2010). https://doi.org/10.1088/0004-637X/721/2/1861

111. Beatty, T.G., Marley, M.S., Gaudi, B.S., Colón, K.D., Fortney, J.J., Showman, A.P.: Spitzer Phase curves of KELT-1b and the signatures of nightside clouds in thermal phase observations. AJ 158(4), 166 (2019). https://doi.org/10.3847/1538-3881/ab33fc

112. Holczer, T., Mazeh, T., Nachmani, G., Jontof-Hutter, D., Ford, E.B., Fabrycky, D., Ragozzine, D., Kane, M., Steffen, J.H.: Transit timing observations from Kepler. IX. Catalog of the full long-cadence data set. Astrophys. J. Suppl. 225(1), 9 (2016). https://doi.org/10.3847/0067-0049/225/1/9

113. Collins, K.A., Kielkopf, J.F., Stassun, K.G.: Transit timing variation measurements of WASP-12b and Qatar-1b: No evidence of additional planets. AJ 153(2), 78 (2017). https://doi.org/10.3847/1538-3881/153/2/78

114. Močnik, T., Southworth, J., Hellier, C.: Recurring sets of recurring starspot occultations on exoplanet host Qatar-2. MNRAS 471(1), 394 (2017). https://doi.org/10.1093/mnras/stx1557

115. Jiang, I.G., Yeh, L.C., Thakur, P., Wu, Y.T., Chien, P., Lin, Y.L., Chen, H.Y., Hu, J.H., Sun, Z., Ji, J.: Possible transit timing variations of the TrES-3 planetary system. AJ 145(3), 68 (2013). https://doi.org/10.1088/0004-6256/145/3/68 
116. Sozzetti, A., Bonomo, A.S., Biazzo, K., Mancini, L., Damasso, M., Desidera, S., Gratton, R., Lanza, A.F., Poretti, E., Rainer, M., Malavolta, L., Affer, L., Barbieri, M., Bedin, L.R., Boccato, C., Bonavita, M., Borsa, F., Ciceri, S., Claudi, R.U., Gandolfi, D., Giacobbe, P., Henning, T., Knapic, C., Latham, D.W., Lodato, G., Maggio, A., Maldonado, J., Marzari, F., Martinez Fiorenzano, A.F., Micela, G., Molinari, E., Mordasini, C., Nascimbeni, V., Pagano, I., Pedani, M., Pepe, F., Piotto, G., Santos, N., Scandariato, G., Shkolnik, E., Southworth, J.: The GAPS programme with HARPS-N at TNG. VI. The curious case of TrES-4b. Astron. Astrophys. 575, L15 (2015). https://doi.org/10.1051/0004-6361/201425570

117. Maciejewski, G., Ohlert, J., Dimitrov, D., Puchalski, D., Nedoroscik, J., Vanko, M., Marka, C., Baar, S., Raetz, S., Seeliger, M., Neuhauser, R.: Revisiting parameters for the WASP-1 planetary system. ACTA Astron. 64(1), 11 (2014)

118. Wong, I., Knutson, H.A., Kataria, T., Lewis, N.K., Burrows, A., Fortney, J.J., Schwartz, J., Shporer, A., Agol, E., Cowan, N.B., Deming, D., Désert, J.M., Fulton, B.J., Howard, A.W., Langton, J., Laughlin, G., Showman, A.P., Todorov, K.: 3.6 and $4.5 \mu \mathrm{m}$ Spitzer phase curves of the highly irradiated hot Jupiters WASP-19b and HAT-P-7b. ApJ 823(2), 122 (2016). https://doi.org/10.3847/0004-637X/823/2/122

119. Southworth, J., Hinse, T.C., Burgdorf, M., Calchi Novati, S., Dominik, M., Galianni, P., Gerner, T., Giannini, E., Gu, S.H., Hundertmark, M., Jørgensen, U.G., Juncher, D., Kerins, E., Mancini, L., Rabus, M., Ricci, D., Schäfer, S., Skottfelt, J., Tregloan-Reed, J., Wang, X.B., Wertz, O., Alsubai, K.A., Andersen, J.M., Bozza, V., Bramich, D.M., Browne, P., Ciceri, S., D’Ago, G., Damerdji, Y., Diehl, C., Dodds, P., Elyiv, A., Fang, X.S., Finet, F., Figuera Jaimes, R., Hardis, S., Harpsoe, K., Jessen-Hansen, J., Kains, N., Kjeldsen, H., Korhonen, H., Liebig, C., Lund, M.N., Lundkvist, M., Mathiasen, M., Penny, M.T., Popovas, A., Proft, S., Rahvar, S., Sahu, K., Scarpetta, G., Schmidt, R.W., Schönebeck, F., Snodgrass, C., Street, R.A., Surdej, J., Tsapras, Y., Vilela, C.: High-precision photometry by telescope defocussing - VI. WASP-24, WASP-25 and WASP-26. MNRAS 444(1), 776 (2014). https://doi.org/10.1093/mnras/stu1492

120. Mancini, L., Kemmer, J., Southworth, J., Bott, K., Mollière, P., Ciceri, S., Chen, G., Henning, T.: An optical transmission spectrum of the giant planet WASP-36 b. MNRAS 459(2), 1393 (2016). https://doi.org/10.1093/mnras/stw659

121. Hoyer, S., Pallé, E., Dragomir, D., Murgas, F.: Ruling out the orbital decay of the WASP-43b exoplanet. AJ 151(6), 137 (2016). https://doi.org/10.3847/0004-6256/151/6/137

122. Ciceri, S., Mancini, L., Southworth, J., Bruni, I., Nikolov, N., D’Ago, G., Schröder, T., Bozza, V., Tregloan-Reed, J., Henning, T.: Physical properties of the HAT-P-23 and WASP48 planetary systems from multi-colour photometry. Astron. Astrophys. 577, A54 (2015). https://doi.org/10.1051/0004-6361/201425449

123. Wyttenbach, A., Lovis, C., Ehrenreich, D., Bourrier, V., Pino, L., Allart, R., Astudillo-Defru, N., Cegla, H.M., Heng, K., Lavie, B., Melo, C., Murgas, F., Santerne, A., Ségransan, D., Udry, S., Pepe, F.: Hot exoplanet atmospheres resolved with transit spectroscopy (HEARTS). I. Detection of hot neutral sodium at high altitudes on WASP-49b. Astron. Astrophys. 602, A36 (2017). https://doi.org/10.1051/0004-6361/201630063

124. Tregloan-Reed, J., Southworth, J.: An extremely high photometric precision in ground-based observations of two transits in the WASP-50 planetary system. MNRAS 431(1), 966 (2013). https://doi.org/10.1093/mnras/stt227

125. Öztürk, O., Erdem, A.: New photometric analysis of five exoplanets: CoRoT-2b, HAT-P-12b, TrES2b, WASP-12b, and WASP-52b. MNRAS 486(2), 2290 (2019). https://doi.org/10.1093/mnras/stz747

126. Southworth, J., Mancini, L., Tregloan-Reed, J., Calchi Novati, S., Ciceri, S., D’Ago, G., Delrez, L., Dominik, M., Evans, D.F., Gillon, M., Jehin, E., Jørgensen, U.G., Haugbólle, T., Lendl, M., Arena, C., Barbieri, L., Barbieri, M., Corfini, G., Lopresti, C., Marchini, A., Marino, G., Alsubai, K.A., Bozza, V., Bramich, D.M., Jaimes, R.F., Hinse, T.C., Henning, T., Hundertmark, M., Juncher, D., Korhonen, H., Popovas, A., Rabus, M., Rahvar, S., Schmidt, R.W., Skottfelt, J., Snodgrass, C., Starkey, D., Surdej, J., Wertz, O.: Larger and faster: revised properties and a shorter orbital period for the WASP-57 planetary system from a pro-am collaboration. MNRAS 454(3), 3094 (2015). https://doi.org/10.1093/mnras/stv2183

127. Triaud, A.H.M.J., Gillon, M., Ehrenreich, D., Herrero, E., Lendl, M., Anderson, D.R., Collier Cameron, A., Delrez, L., Demory, B.O., Hellier, C., Heng, K., Jehin, E., Maxted, P.F.L., Pollacco, D., Queloz, D., Ribas, I., Smalley, B., Smith, A.M.S., Udry, S.: WASP-80b has a dayside within the T-dwarf range. MNRAS 450(3), 2279 (2015). https://doi.org/10.1093/mnras/stv706 
128. Močnik, T., Clark, B.J.M., Anderson, D.R., Hellier, C., Brown, D.J.A.: Starspots on WASP-85. AJ 151(6), 150 (2016). https://doi.org/10.3847/0004-6256/151/6/150

129. Hay, K.L., Collier-Cameron, A., Doyle, A.P., Hébrard, G., Skillen, I., Anderson, D.R., Barros, S.C.C., Brown, D.J.A., Bouchy, F., Busuttil, R., Delorme, P., Delrez, L., Demangeon, O., Díaz, R.F., Gillon, M., Gómez Maqueo Chew, Y., Gonzàlez, E., Hellier, C., Holmes, S., Jarvis, J.F., Jehin, E., Joshi, Y.C., Kolb, U., Lendl, M., Maxted, P.F.L., McCormac, J., Miller, G.R.M., Mortier, A., Pallé, E., Pollacco, D., Prieto-Arranz, J., Queloz, D., Ségransan, D., Simpson, E.K., Smalley, B., Southworth, J., Triaud, A.H.M.J., Turner, O.D., Udry, S., Vanhuysse, M., West, R.G., Wilson, P.A.: WASP-92b, WASP-93b and WASP-118b: three new transiting close-in giant planets. MNRAS 463(3), 3276 (2016). https://doi.org/10.1093/mnras/stw2090

130. Southworth, J., Mancini, L., Ciceri, S., Budaj, J., Dominik, M., Figuera Jaimes, R., Haugbølle, T., Jørgensen, U.G., Popovas, A., Rabus, M., Rahvar, S., von Essen, C., Schmidt, R.W., Wertz, O., Alsubai, K.A., Bozza, V., Bramich, D.M., Calchi Novati, S., D’Ago, G., Hinse, T.C., Henning, T., Hundertmark, M., Juncher, D., Korhonen, H., Skottfelt, J., Snodgrass, C., Starkey, D., Surdej, J.: High-precision photometry by telescope defocusing - VII. The ultrashort period planet WASP-103. MNRAS 447(1), 711 (2015). https://doi.org/10.1093/mnras/stu2394

131. Barros, S.C.C., Brown, D.J.A., Hébrard, G., Gómez Maqueo Chew, Y., Anderson, D.R., Boumis, P., Delrez, L., Hay, K.L., Lam, K.W.F., Llama, J., Lendl, M., McCormac, J., Skiff, B., Smalley, B., Turner, O., Vanhuysse, M., Armstrong, D.J., Boisse, I., Bouchy, F., Collier Cameron, A., Faedi, F., Gillon, M., Hellier, C., Jehin, E., Liakos, A., Meaburn, J., Osborn, H.P., Pepe, F., Plauchu-Frayn, I., Pollacco, D., Queloz, D., Rey, J., Spake, J., Ségransan, D., Triaud, A.H.M., Udry, S., Walker, S.R., Watson, C.A., West, R.G., Wheatley, P.J.: WASP-113b and WASP114b, two inflated hot Jupiters with contrasting densities. Astron. Astrophys. 593, A113 (2016). https://doi.org/10.1051/0004-6361/201526517

132. Demangeon, O.D.S., Faedi, F., Hébrard, G., Brown, D.J.A., Barros, S.C.C., Doyle, A.P., Maxted, P.F.L., Collier Cameron, A., Hay, K.L., Alikakos, J., Anderson, D.R., Armstrong, D.J., Boumis, P., Bonomo, A.S., Bouchy, F., Delrez, L., Gillon, M., Haswell, C.A., Hellier, C., Jehin, E., Kiefer, F., Lam, K.W.F., Lendl, M., Mancini, L., McCormac, J., Norton, A.J., Osborn, H.P., Palle, E., Pepe, F., Pollacco, D.L., Prieto-Arranz, J., Queloz, D., Ségransan, D., Smalley, B., Triaud, A.H.M.J., Udry, S., West, R., Wheatley, P.J.: The discovery of WASP-151b, WASP-153b, WASP-156b: Insights on giant planet migration and the upper boundary of the Neptunian desert. Astron. Astrophys. 610, A63 (2018). https://doi.org/10.1051/0004-6361/201731735

133. Burke, C.J., McCullough, P.R., Bergeron, L.E., Long, D., Gilliland, R.L., Nelan, E.P., Johns-Krull, C.M., Valenti, J.A., Janes, K.A.: NICMOS observations of the transiting hot Jupiter XO-1b. ApJ 719(2), 1796 (2010). https://doi.org/10.1088/0004-637X/719/2/1796

134. Damasso, M., Biazzo, K., Bonomo, A.S., Desidera, S., Lanza, A.F., Nascimbeni, V., Esposito, M., Scand ariato, G., Sozzetti, A., Cosentino, R., Gratton, R., Malavolta, L., Rainer, M., Gandolfi, D., Poretti, E., Zanmar Sanchez, R., Ribas, I., Santos, N., Affer, L., Andreuzzi, G., Barbieri, M., Bedin, L.R., Benatti, S., Bernagozzi, A., Bertolini, E., Bonavita, M., Borsa, F., Borsato, L., Boschin, W., Calcidese, P., Carbognani, A., Cenadelli, D., Christille, J.M., Claudi, R.U., Covino, E., Cunial, A., Giacobbe, P., Granata, V., Harutyunyan, A., Lattanzi, M.G., Leto, G., Libralato, M., Lodato, G., Lorenzi, V., Mancini, L., Martinez Fiorenzano, A.F., Marzari, F., Masiero, S., Micela, G., Molinari, E., Molinaro, M., Munari, U., Murabito, S., Pagano, I., Pedani, M., Piotto, G., Rosenberg, A., Silvotti, R., Southworth, J.: The GAPS programme with HARPS-N at TNG. V. A comprehensive analysis of the XO-2 stellar and planetary systems. Astron. Astrophys. 575, A111 (2015). https://doi.org/10.1051/0004-6361/201425332

135. Smith, A.M.S.: The properties of XO-5b and WASP-82b redetermined using new high-precision transit photometry and global data analyses. ACTA Astron. 65, 117 (2015)

136. Chavero, C., de La Reza, R., Domingos, R.C., Drake, N.A., Pereira, C.B., Winter, O.C.: Distribution of refractory and volatile elements in CoRoT exoplanet host stars. Astron. Astrophys. 517, A40 (2010). https://doi.org/10.1051/0004-6361/200912184

137. Alonso, R., Auvergne, M., Baglin, A., Ollivier, M., Moutou, C., Rouan, D., Deeg, H.J., Aigrain, S., Almenara, J.M., Barbieri, M., Barge, P., Benz, W., Bordé, P., Bouchy, F., de La Reza, R., Deleuil, M., Dvorak, R., Erikson, A., Fridlund, M., Gillon, M., Gondoin, P., Guillot, T., Hatzes, A., Hébrard, G., Kabath, P., Jorda, L., Lammer, H., Léger, A., Llebaria, A., Loeillet, B., Magain, P., Mayor, M., Mazeh, T., Pätzold, M., Pepe, F., Pont, F., Queloz, D., Rauer, H., Shporer, A., Schneider, J., Stecklum, B., Udry, S., Wuchterl, G.: Transiting exoplanets from the CoRoT space mission. II. 
CoRoT-Exo-2b: a transiting planet around an active G star. Astron. Astrophys. 482(3), L21 (2008). https://doi.org/10.1051/0004-6361:200809431

138. Hartman, J.D., Bakos, G.Á. ., Bhatti, W., Penev, K., Bieryla, A., Latham, D.W., Kovács, G., Torres, G., Csubry, Z., de Val-Borro, M., Buchhave, L., Kovács, T., Quinn, S., Howard, A.W., Isaacson, H., Fulton, B.J., Everett, M.E., Esquerdo, G., Béky, B., Szklenar, T., Falco, E., Santerne, A., Boisse, I., Hébrard, G., Burrows, A., Lázár, J., Papp, I., Sári, P.: HAT-P-65b and HAT-P-66b: Two transiting inflated hot Jupiters and observational evidence for the reinflation of close-in giant planets. AJ 152(6), 182 (2016). https://doi.org/10.3847/0004-6256/152/6/182

139. Smalley, B., Anderson, D.R., Collier Cameron, A., Gillon, M., Hellier, C., Lister, T.A., Maxted, P.F.L., Queloz, D., Triaud, A.H.M.J., West, R.G., Bentley, S.J., Enoch, B., Pepe, F., Pollacco, D.L., Segransan, D., Smith, A.M.S., Southworth, J., Udry, S., Wheatley, P.J., Wood, P.L., Bento, J.: WASP26b: a 1-Jupiter-mass planet around an early-G-type star. Astron. Astrophys. 520, A56 (2010). https://doi.org/10.1051/0004-6361/201014705

140. Charbonneau, D., Berta, Z.K., Irwin, J., Burke, C.J., Nutzman, P., Buchhave, L.A., Lovis, C., Bonfils, X., Latham, D.W., Udry, S., Murray-Clay, R.A., Holman, M.J., Falco, E.E., Winn, J.N., Queloz, D., Pepe, F., Mayor, M., Delfosse, X., Forveille, T.: A super-Earth transiting a nearby low-mass star. Nature 462(7275), 891 (2009). https://doi.org/10.1038/nature08679

141. Berta, Z.K., Charbonneau, D., Désert, J.M., Miller-Ricci Kempton, E., McCullough, P.R., Burke, C.J., Fortney, J.J., Irwin, J., Nutzman, P., Homeier, D.: The flat transmission spectrum of the superearth GJ1214b from wide field camera 3 on the hubble space telescope. ApJ 747(1), 35 (2012). https://doi.org/10.1088/0004-637X/747/1/35

142. Hartman, J.D., Bayliss, D., Brahm, R., Bakos, G.Á.., Mancini, L., Jordán, A., Penev, K., Rabus, M., Zhou, G., Butler, R.P., Espinoza, N., de Val-Borro, M., Bhatti, W., Csubry, Z., Ciceri, S., Henning, T., Schmidt, B., Arriagada, P., Shectman, S., Crane, J., Thompson, I., Suc, V., Csák, B., Tan, T.G., Noyes, R.W., Lázár, J., Papp, I., Sári, P.: HATS-6b: A warm saturn transiting an early M dwarf star, and a set of empirical relations for characterizing $\mathrm{K}$ and M dwarf planet hosts. AJ 149(5), 166 (2015). https://doi.org/10.1088/0004-6256/149/5/166

143. Torres, G., Winn, J.N., Holman, M.J.: Improved parameters for extrasolar transiting planets. ApJ 677(2), 1324 (2008). https://doi.org/10.1086/529429

144. Knutson, H.A., Madhusudhan, N., Cowan, N.B., Christiansen, J.L., Agol, E., Deming, D., Désert, J.M., Charbonneau, D., Henry, G.W., Homeier, D., Langton, J., Laughlin, G., Seager, S.: A Spitzer transmission spectrum for the exoplanet GJ 436b, evidence for stellar variability, and constraints on dayside flux variations. ApJ 735(1), 27 (2011). https://doi.org/10.1088/0004-637X/735/1/27

145. Bento, J., Schmidt, B., Hartman, J.D., Bakos, G.Á.., Ciceri, S., Brahm, R., Bayliss, D., Espinoza, N., Zhou, G., Rabus, M., Bhatti, W., Penev, K., Csubry, Z., Jordán, A., Mancini, L., Henning, T., de Val-Borro, M., Tinney, C.G., Wright, D.J., Durkan, S., Suc, V., Noyes, R., Lázár, J., Papp, I., Sári, P.: HATS-22b, HATS-23b and HATS-24b: three new transiting super-Jupiters from the HATSouth project. MNRAS 468(1), 835 (2017). https://doi.org/10.1093/mnras/stx500

146. Espinoza, N., Bayliss, D., Hartman, J.D., Bakos, G.Á.., Jordán, A., Zhou, G., Mancini, L., Brahm, R., Ciceri, S., Bhatti, W., Csubry, Z., Rabus, M., Penev, K., Bento, J., de Val-Borro, M., Henning, T., Schmidt, B., Suc, V., Wright, D.J., Tinney, C.G., Tan, T.G., Noyes, R.: HATS-25b through HATS30b: A half-dozen new inflated transiting hot Jupiters from the HATSouth survey. AJ 152(4), 108 (2016). https://doi.org/10.3847/0004-6256/152/4/108

147. Smith, A.M.S., Anderson, D.R., Collier Cameron, A., Gillon, M., Hellier, C., Lendl, M., Maxted, P.F.L., Queloz, D., Smalley, B., Triaud, A.H.M.J., West, R.G., Barros, S.C.C., Jehin, E., Pepe, F., Pollacco, D., Segransan, D., Southworth, J., Street, R.A., Udry, S.: WASP-36b: A new transiting planet around a metal-poor G-dwarf, and an investigation into analyses based on a single transit light curve. AJ 143(4), 81 (2012). https://doi.org/10.1088/0004-6256/143/4/81

148. Morello, G., Waldmann, I.P., Tinetti, G., Peres, G., Micela, G., Howarth, I.D.: A new look at Spitzer primary transit observations of the Exoplanet HD 189733b. ApJ 786(1), 22 (2014). https://doi.org/10.1088/0004-637X/786/1/22

149. Hellier, C., Anderson, D.R., Collier Cameron, A., Gillon, M., Jehin, E., Lendl, M., Maxted, P.F.L., Pepe, F., Pollacco, D., Queloz, D., Ségransan, D., Smalley, B., Smith, A.M.S., Southworth, J., Triaud, A.H.M.J., Udry, S., West, R.G.: WASP-43b: the closest-orbiting hot Jupiter. Astron. Astrophys. 535, L7 (2011). https://doi.org/10.1051/0004-6361/201117081 
150. Knutson, H.A., Charbonneau, D., Noyes, R.W., Brown, T.M., Gilliland, R.L.: Using stellar limb-darkening to refine the properties of HD 209458b. ApJ 655(1), 564 (2007). https://doi.org/10.1086/510111

151. Mortier, A., Santos, N.C., Sousa, S.G., Fernand es, J.M., Adibekyan, V.Z., Delgado Mena, E., Montalto, M., Israelian, G.: New and updated stellar parameters for 90 transit hosts. The effect of the surface gravity. Astron. Astrophys. 558, A106 (2013). https://doi.org/10.1051/0004-6361/201322240

152. Hébrard, G., Désert, J.M., Díaz, R.F., Boisse, I., Bouchy, F., Lecavelier Des Etangs, A., Moutou, C., Ehrenreich, D., Arnold, L., Bonfils, X., Delfosse, X., Desort, M., Eggenberger, A., Forveille, T., Gregorio, J., Lagrange, A.M., Lovis, C., Pepe, F., Perrier, C., Pont, F., Queloz, D., Santerne, A., Santos, N.C., Ségransan, D., Sing, D.K., Udry, S., Vidal-Madjar, A.: Observation of the full 12hour-long transit of the exoplanet HD 80606b. Warm-Spitzer photometry and SOPHIE spectroscopy. Astron. Astrophys. 516, A95 (2010). https://doi.org/10.1051/0004-6361/201014327

153. Lendl, M., Anderson, D.R., Collier-Cameron, A., Doyle, A.P., Gillon, M., Hellier, C., Jehin, E., Lister, T.A., Maxted, P.F.L., Pepe, F., Pollacco, D., Queloz, D., Smalley, B., Ségransan, D., Smith, A.M.S., Triaud, A.H.M.J., Udry, S., West, R.G., Wheatley, P.J.: WASP-42 b and WASP-49 b: two new transiting sub-Jupiters. Astron. Astrophys. 544, A72 (2012). https://doi.org/10.1051/0004-6361/201219585

154. Noyes, R.W., Bakos, G.Á.., Torres, G., Pál, A., Kovács, G., Latham, D.W., Fernández, J.M., Fischer, D.A., Butler, R.P., Marcy, G.W., Sipócz, B., Esquerdo, G.A., Kovács, G., Sasselov, D.D., Sato, B., Stefanik, R., Holman, M., Lázár, J., Papp, I., Sári, P.: HAT-P-6b: A hot jupiter transiting a bright F star. Astrophys. J. Lett. 673(1), L79 (2008). https://doi.org/10.1086/527358

155. Santerne, A., Hébrard, G., Lillo-Box, J., Armstrong, D.J., Barros, S.C.C., Demangeon, O., Barrado, D., Debackere, A., Deleuil, M., Delgado Mena, E., Montalto, M., Pollacco, D., Osborn, H.P., Sousa, S.G., Abe, L., Adibekyan, V., Almenara, J.M., André, P., Arlic, G., Barthe, G., Bendjoya, P., Behrend, R., Boisse, I., Bouchy, F., Boussier, H., Bretton, M., Brown, D.J.A., Carry, B., Cailleau, A., Conseil, E., Coulon, G., Courcol, B., Dauchet, B., Dalouzy, J.C., Deldem, M., Desormières, O., Dubreuil, P., Fehrenbach, J.M., Ferratfiat, S., Girelli, R., Gregorio, J., Jaecques, S., Kugel, F., Kirk, J., Labrevoir, O., Lachurié, J.C., Lam, K.W.F., Le Guen, P., Martinez, P., Maurin, L.P.A., McCormac, J., Pioppa, J.B., Quadri, U., Rajpurohit, A., Rey, J., Rivet, J.P., Roy, R., Santos, N.C., Signoret, F., Strabla, L., Suarez, O., Toublanc, D., Tsantaki, M., Vienney, J.M., Wilson, P.A., Bachschmidt, M., Colas, F., Gerteis, O., Louis, P., Mario, J.C., Marlot, C., Montier, J., Perroud, V., Pic, V., Romeuf, D., Ubaud, S., Verilhac, D.: K2-29 b/WASP-152 b: An aligned and inflated hot jupiter in a young visual binary. ApJ 824(1), 55 (2016). https://doi.org/10.3847/0004-637X/824/1/55

156. Gillon, M., Doyle, A.P., Lendl, M., Maxted, P.F.L., Triaud, A.H.M.J., Anderson, D.R., Barros, S.C.C., Bento, J., Collier-Cameron, A., Enoch, B., Faedi, F., Hellier, C., Jehin, E., Magain, P., Montalbán, J., Pepe, F., Pollacco, D., Queloz, D., Smalley, B., Segransan, D., Smith, A.M.S., Southworth, J., Udry, S., West, R.G., Wheatley, P.J.: WASP-50 b: a hot Jupiter transiting a moderately active solar-type star. Astron. Astrophys. 533, A88 (2011). https://doi.org/10.1051/0004-6361/201117198

157. Pál, A., Bakos, G.Á.., Torres, G., Noyes, R.W., Latham, D.W., Kovács, G., Marcy, G.W., Fischer, D.A., Butler, R.P., Sasselov, D.D., Sipócz, B., Esquerdo, G.A., Kovács, G., Stefanik, R., Lázár, J., Papp, I., Sári, P.: HAT-P-7b: An extremely hot massive planet transiting a bright star in the kepler field. ApJ 680(2), 1450 (2008). https://doi.org/10.1086/588010

158. Hébrard, G., Collier Cameron, A., Brown, D.J.A., Díaz, R.F., Faedi, F., Smalley, B., Anderson, D.R., Armstrong, D., Barros, S.C.C., Bento, J., Bouchy, F., Doyle, A.P., Enoch, B., Gómez Maqueo Chew, Y., Hébrard, É. M., Hellier, C., Lendl, M., Lister, T.A., Maxted, P.F.L., McCormac, J., Moutou, C., Pollacco, D., Queloz, D., Santerne, A., Skillen, I., Southworth, J., Tregloan-Reed, J., Triaud, A.H.M.J., Udry, S., Vanhuysse, M., Watson, C.A., West, R.G., Wheatley, P.J.: WASP-52b, WASP58b, WASP-59b, and WASP-60b: Four new transiting close-in giant planets. Astron. Astrophys. 549, A134 (2013). https://doi.org/10.1051/0004-6361/201220363

159. Latham, D.W., Bakos, G.Á.., Torres, G., Stefanik, R.P., Noyes, R.W., Kovács, G., Pál, A., Marcy, G.W., Fischer, D.A., Butler, R.P., Sipócz, B., Sasselov, D.D., Esquerdo, G.A., Vogt, S.S., Hartman, J.D., Kovács, G., Lázár, J., Papp, I., Sári, P.: Discovery of a transiting planet and eight eclipsing binaries in HATNet field G205. ApJ 704(2), 1107 (2009). https://doi.org/10.1088/0004-637X/704/2/1107

160. Mancini, L., Southworth, J., Ciceri, S., Fortney, J.J., Morley, C.V., Dittmann, J.A., Tregloan-Reed, J., Bruni, I., Barbieri, M., Evans, D.F., D’Ago, G., Nikolov, N., Henning, T.: A lower radius and mass for the transiting extrasolar planet HAT-P-8 b. Astron. Astrophys. 551, A11 (2013). https://doi.org/10.1051/0004-6361/201220291 
161. Siverd, R.J., Beatty, T.G., Pepper, J., Eastman, J.D., Collins, K., Bieryla, A., Latham, D.W., Buchhave, L.A., Jensen, E.L.N., Crepp, J.R., Street, R., Stassun, K.G., Gaudi, B.S., Berlind, P., Calkins, M.L., DePoy, D.L., Esquerdo, G.A., Fulton, B.J., Fưrész, G., Geary, J.C., Gould, A., Hebb, L., Kielkopf, J.F., Marshall, J.L., Pogge, R., Stanek, K.Z., Stefanik, R.P., Szentgyorgyi, A.H., Trueblood, M., Trueblood, P., Stutz, A.M., van Saders, J.L.: KELT-1b: A strongly irradiated, highly inflated, short period, 27 Jupiter-mass companion transiting a mid-F star. ApJ 761(2), 123 (2012). https://doi.org/10.1088/0004-637X/761/2/123

162. Southworth, J., Tregloan-Reed, J., Andersen, M.I., Calchi Novati, S., Ciceri, S., Colque, J.P., D’Ago, G., Dominik, M., Evans, D.F., Gu, S.H., Herrera-Cordova, A., Hinse, T.C., Jørgensen, U.G., Juncher, D., Kuffmeier, M., Mancini, L., Peixinho, N., Popovas, A., Rabus, M., Skottfelt, J., Tronsgaard, R., Unda-Sanzana, E., Wang, X.B., Wertz, O., Alsubai, K.A., Andersen, J.M., Bozza, V., Bramich, D.M., Burgdorf, M., Damerdji, Y., Diehl, C., Elyiv, A., Figuera Jaimes, R., Haugbølle, T., Hundertmark, M., Kains, N., Kerins, E., Korhonen, H., Liebig, C., Mathiasen, M., Penny, M.T., Rahvar, S., Scarpetta, G., Schmidt, R.W., Snodgrass, C., Starkey, D., Surdej, J., Vilela, C., von Essen, C., Wang, Y.: High-precision photometry by telescope defocussing - VIII. WASP-22, WASP-41, WASP-42 and WASP-55. MNRAS 457(4), 4205 (2016). https://doi.org/10.1093/mnras/stw279

163. Shporer, A., Bakos, G.Á.., Bouchy, F., Pont, F., Kovács, G., Latham, D.W., Sipöcz, B., Torres, G., Mazeh, T., Esquerdo, G.A., Pál, A., Noyes, R.W., Sasselov, D.D., Lázár, J., Papp, I., Sári, P., Kovács, G.: HAT-P-9b: A low-density planet transiting a moderately faint F star. ApJ 690(2), 1393 (2009). https://doi.org/10.1088/0004-637X/690/2/1393

164. Pepper, J., Siverd, R.J., Beatty, T.G., Gaudi, B.S., Stassun, K.G., Eastman, J., Collins, K., Latham, D.W., Bieryla, A., Buchhave, L.A., Jensen, E.L.N., Manner, M., Penev, K., Crepp, J.R., Cargile, P.A., Dhital, S., Calkins, M.L., Esquerdo, G.A., Berlind, P., Fulton, B.J., Street, R., Ma, B., Ge, J., Wang, J., Mao, Q., Richert, A.J.W., Gould, A., DePoy, D.L., Kielkopf, J.F., Marshall, J.L., Pogge, R.W., Stefanik, R.P., Trueblood, M., Trueblood, P.: KELT-3b: A hot Jupiter transiting a V = 9.8 Late-F Star. ApJ 773(1), 64 (2013). https://doi.org/10.1088/0004-637X/773/1/64

165. Faedi, F., Pollacco, D., Barros, S.C.C., Brown, D., Collier Cameron, A., Doyle, A.P., Enoch, R., Gillon, M., Gómez Maqueo Chew, Y., Hébrard, G., Lendl, M., Liebig, C., Smalley, B., Triaud, A.H.M.J., West, R.G., Wheatley, P.J., Alsubai, K.A., Anderson, D.R., Armstrong, D., Bento, J., Bochinski, J., Bouchy, F., Busuttil, R., Fossati, L., Fumel, A., Haswell, C.A., Hellier, C., Holmes, S., Jehin, E., Kolb, U., McCormac, J., Miller, G.R.M., Moutou, C., Norton, A.J., Parley, N., Queloz, D., Santerne, A., Skillen, I., Smith, A.M.S., Udry, S., Watson, C.: WASP-54b, WASP-56b, and WASP57b: Three new sub-Jupiter mass planets from SuperWASP. Astron. Astrophys. 551, A73 (2013). https://doi.org/10.1051/0004-6361/201220520

166. Hartman, J.D., Bakos, G.Á.., Torres, G., Kovács, G., Noyes, R.W., Pál, A., Latham, D.W., Sipócz, B., Fischer, D.A., Johnson, J.A., Marcy, G.W., Butler, R.P., Howard, A.W., Esquerdo, G.A., Sasselov, D.D., Kovács, G., Stefanik, R.P., Fernandez, J.M., Lázár, J., Papp, I., Sári, P.: HAT-P-12b: A low-density sub-saturn mass planet transiting a metal-poor K dwarf. ApJ 706(1), 785 (2009). https://doi.org/10.1088/0004-637X/706/1/785

167. Bakos, G.Á.., Howard, A.W., Noyes, R.W., Hartman, J., Torres, G., Kovács, G., Fischer, D.A., Latham, D.W., Johnson, J.A., Marcy, G.W., Sasselov, D.D., Stefanik, R.P., Sipócz, B., Kovács, G., Esquerdo, G.A., Pál, A., Lázár, J., Papp, I., Sári, P.: HAT-P-13b,c: A transiting hot jupiter with a massive outer companion on an eccentric orbit. ApJ 707(1), 446 (2009). https://doi.org/10.1088/0004-637X/707/1/446

168. Fulton, B.J., Collins, K.A., Gaudi, B.S., Stassun, K.G., Pepper, J., Beatty, T.G., Siverd, R.J., Penev, K., Howard, A.W., Baranec, C., Corfini, G., Eastman, J.D., Gregorio, J., Law, N.M., Lund, M.B., Oberst, T.E., Penny, M.T., Riddle, R., Rodriguez, J.E., Stevens, D.J., Zambelli, R., Ziegler, C., Bieryla, A., D’Ago, G., DePoy, D.L., Jensen, E.L.N., Kielkopf, J.F., Latham, D.W., Manner, M., Marshall, J., McLeod, K.K., Reed, P.A.: KELT-8b: A highly inflated transiting hot jupiter and a new technique for extracting high-precision radial velocities from noisy spectra. ApJ 810(1), 30 (2015). https://doi.org/10.1088/0004-637X/810/1/30

169. Buchhave, L.A., Bakos, G.Á.., Hartman, J.D., Torres, G., Kovács, G., Latham, D.W., Noyes, R.W., Esquerdo, G.A., Everett, M., Howard, A.W., Marcy, G.W., Fischer, D.A., Johnson, J.A., Andersen, J., Fúrész, G., Perumpilly, G., Sasselov, D.D., Stefanik, R.P., Béky, B., Lázár, J., Papp, I., Sári, P.: HAT-P-16b: A $4 \mathrm{M}_{J}$ planet transiting a bright star on an eccentric orbit. ApJ 720(2), 1118 (2010). https://doi.org/10.1088/0004-637X/720/2/1118 
170. Howard, A.W., Bakos, G.Á.., Hartman, J., Torres, G., Shporer, A., Mazeh, T., Kovács, G., Latham, D.W., Noyes, R.W., Fischer, D.A., Johnson, J.A., Marcy, G.W., Esquerdo, G.A., Béky, B., Butler, R.P., Sasselov, D.D., Stefanik, R.P., Perumpilly, G., Lázár, J., Papp, I., Sári, P.: HAT-P-17b,c: A transiting, eccentric, hot saturn and a long-period, cold Jupiter. ApJ 749(2), 134 (2012). https://doi.org/10.1088/0004-637X/749/2/134

171. Anderson, D.R., Collier Cameron, A., Delrez, L., Doyle, A.P., Faedi, F., Fumel, A., Gillon, M., Gómez Maqueo Chew, Y., Hellier, C., Jehin, E., Lendl, M., Maxted, P.F.L., Pepe, F., Pollacco, D., Queloz, D., Ségransan, D., Skillen, I., Smalley, B., Smith, A.M.S., Southworth, J., Triaud, A.H.M.J., Turner, O.D., Udry, S., West, R.G.: Three newly discovered sub-Jupiter-mass planets: WASP-69b and WASP-84b transit active K dwarfs and WASP-70Ab transits the evolved primary of a G4+K3 binary. MNRAS 445(2), 1114 (2014). https://doi.org/10.1093/mnras/stu1737

172. Hartman, J.D., Bakos, G.Á.., Sato, B., Torres, G., Noyes, R.W., Latham, D.W., Kovács, G., Fischer, D.A., Howard, A.W., Johnson, J.A., Marcy, G.W., Buchhave, L.A., Füresz, G., Perumpilly, G., Béky, B., Stefanik, R.P., Sasselov, D.D., Esquerdo, G.A., Everett, M., Csubry, Z., Lázár, J., Papp, I., Sári, P.: HAT-P-18b and HAT-P-19b: Two low-density saturn-mass planets transiting metal-rich K Stars. ApJ 726(1), 52 (2011). https://doi.org/10.1088/0004-637X/726/1/52

173. Kirk, J., Wheatley, P.J., Louden, T., Doyle, A.P., Skillen, I., McCormac, J., Irwin, P.G.J., Karjalainen, R.: Rayleigh scattering in the transmission spectrum of HAT-P-18b. MNRAS 468(4), 3907 (2017). https://doi.org/10.1093/mnras/stx752

174. Dunham, E.W., Borucki, W.J., Koch, D.G., Batalha, N.M., Buchhave, L.A., Brown, T.M., Caldwell, D.A., Cochran, W.D., Endl, M., Fischer, D., Fűrész, G., Gautier, I., Thomas, N., Geary, J.C., Gilliland, R.L., Gould, A., Howell, S.B., Jenkins, J.M., Kjeldsen, H., Latham, D.W., Lissauer, J.J., Marcy, G.W., Meibom, S., Monet, D.G., Rowe, J.F., Sasselov, D.D.: Kepler-6b: A transiting hot jupiter orbiting a metal-rich star. Astrophys. J. Lett. 713(2), L136 (2010). https://doi.org/10.1088/2041-8205/713/2/L136

175. Esteves, L.J., De Mooij, E.J.W., Jayawardhana, R.: Changing phases of alien worlds: Probing atmospheres of kepler planets with high-precision photometry. ApJ 804(2), 150 (2015). https://doi.org/10.1088/0004-637X/804/2/150

176. Alsubai, K.A., Parley, N.R., Bramich, D.M., West, R.G., Sorensen, P.M., Collier Cameron, A., Latham, D.W., Horne, K., Anderson, D.R., Bakos, G.Á.., Brown, D.J.A., Buchhave, L.A., Esquerdo, G.A., Everett, M.E., Fżrész, G., Hartman, J.D., Hellier, C., Miller, G.M., Pollacco, D., Quinn, S.N., Smith, J.C., Stefanik, R.P., Szentgyorgyi, A.: Qatar-1b: a hot Jupiter orbiting a metal-rich K dwarf star. MNRAS 417(1), 709 (2011). https://doi.org/10.1111/j.1365-2966.2011.19316.x

177. West, R.G., Hellier, C., Almenara, J.M., Anderson, D.R., Barros, S.C.C., Bouchy, F., Brown, D.J.A., Collier Cameron, A., Deleuil, M., Delrez, L., Doyle, A.P., Faedi, F., Fumel, A., Gillon, M., Gómez Maqueo Chew, Y., Hébrard, G., Jehin, E., Lendl, M., Maxted, P.F.L., Pepe, F., Pollacco, D., Queloz, D., Ségransan, D., Smalley, B., Smith, A.M.S., Southworth, J., Triaud, A.H.M.J., Udry, S.: Three irradiated and bloated hot Jupiters:. WASP-76b, WASP-82b, and WASP-90b. Astron. Astrophys. 585, A126 (2016). https://doi.org/10.1051/0004-6361/201527276

178. Bryan, M.L., Alsubai, K.A., Latham, D.W., Parley, N.R., Collier Cameron, A., Quinn, S.N., Carter, J.A., Fulton, B.J., Berlind, P., Brown, W.R., Buchhave, L.A., Calkins, M.L., Esquerdo, G.A., Fúrész, G., Gråe Jørgensen, U., Horne, K.D., Stefanik, R.P., Street, R.A., Torres, G., West, R.G., Dominik, M., Harpsœ, K.B.W., Liebig, C., Calchi Novati, S., Ricci, D., Skottfelt, J.F.: Qatar-2: A K dwarf orbited by a transiting hot jupiter and a more massive companion in an outer orbit. ApJ 750(1), 84 (2012). https://doi.org/10.1088/0004-637X/750/1/84

179. Triaud, A.H.M.J., Anderson, D.R., Collier Cameron, A., Doyle, A.P., Fumel, A., Gillon, M., Hellier, C., Jehin, E., Lendl, M., Lovis, C., Maxted, P.F.L., Pepe, F., Pollacco, D., Queloz, D., Ségransan, D., Smalley, B., Smith, A.M.S., Udry, S., West, R.G., Wheatley, P.J.: WASP-80b: a gas giant transiting a cool dwarf. Astron. Astrophys. 551, A80 (2013). https://doi.org/10.1051/0004-6361/201220900

180. Quinn, S.N., Bakos, G.Á. ., Hartman, J., Torres, G., Kovács, G., Latham, D.W., Noyes, R.W., Fischer, D.A., Johnson, J.A., Marcy, G.W., Howard, A.W., Szentgyorgyi, A., Fưrész, G., Buchhave, L.A., Béky, B., Sasselov, D.D., Stefanik, R.P., Perumpilly, G., Everett, M., Lázár, J., Papp, I., Sári, P.: HAT-P-25b: A hot-jupiter transiting a moderately faint G star. ApJ 745(1), 80 (2012). https://doi.org/10.1088/0004-637X/745/1/80

181. Wang, X.Y., Wang, S., Hinse, T.C., Li, K., Wang, Y.H., Laughlin, G., Liu, H.G., Zhang, H., Wu, Z.Y., Zhou, X., Zhou, J.L., Hu, S.M., Wu, D.H., Peng, X.Y., Chen, Y.Y.: Transiting Exoplanet monitoring project (TEMP). IV. Refined system parameters, transit timing variations, and orbital stability 
of the transiting planetary system HAT-P-25. Publ. Astron. Soc. Pac. 130(988), 064401 (2018). https://doi.org/10.1088/1538-3873/aab93e

182. Hartman, J.D., Bakos, G.Á. ., Kipping, D.M., Torres, G., Kovács, G., Noyes, R.W., Latham, D.W., Howard, A.W., Fischer, D.A., Johnson, J.A., Marcy, G.W., Isaacson, H., Quinn, S.N., Buchhave, L.A., Béky, B., Sasselov, D.D., Stefanik, R.P., Esquerdo, G.A., Everett, M., Perumpilly, G., Lázár, J., Papp, I., Sári, P.: HAT-P-26b: A low-density neptune-mass planet transiting a K Star. ApJ 728(2), 138 (2011). https://doi.org/10.1088/0004-637X/728/2/138

183. Stevenson, K.B., Bean, J.L., Seifahrt, A., Gilbert, G.J., Line, M.R., Désert, J.M., Fortney, J.J.: A search for water in the atmosphere of hat-p-26b using LDSS-3C. ApJ 817(2), 141 (2016). https://doi.org/10.3847/0004-637X/817/2/141

184. Brown, D.J.A., Anderson, D.R., Doyle, A.P., Maxted, E.G.F.L., Smalley, B., McCormac, J., Almenera, J.M., Prieto-Arranz, J., Deleuil, M., Diaz, R.F., Foxell, E., Hebrard, G., Lendl, M., Delrez, L., Gillon, M., Jehin, E., Lam, K.W.F., Triaud, A.H.M.J., Turner, O.D., Armstrong, D.J., Bouchy, F., Collier Cameron, A., Pollacco, D., Faedi, F., Gomez Maqueo Chew, Y., Hebb, L., Hellier, C., NeveuVanMalle, M., Palle, E., Queloz, D., Segransan, D., Udry, S.: Three transiting planet discoveries from the Wide Angle Search for Planets: WASP-85 A b; WASP-116 b, and WASP-149 b. arXiv e-prints arXiv:1412.7761 (2014)

185. Mandushev, G., Quinn, S.N., Buchhave, L.A., Dunham, E.W., Rabus, M., Oetiker, B., Latham, D.W., Charbonneau, D., Brown, T.M., Belmonte, J.A., O’Donovan, F.T.: TrES-5: A massive jupiter-sized planet transiting a cool G dwarf. ApJ 741(2), 114 (2011). https://doi.org/10.1088/0004-637X/741/2/ 114

186. Gillon, M., Anderson, D.R., Collier-Cameron, A., Delrez, L., Hellier, C., Jehin, E., Lendl, M., Maxted, P.F.L., Pepe, F., Pollacco, D., Queloz, D., Ségransan, D., Smith, A.M.S., Smalley, B., Southworth, J., Triaud, A.H.M.J., Udry, S., Van Grootel, V., West, R.G.: WASP103 b: a new planet at the edge of tidal disruption. Astron. Astrophys. 562, L3 (2014). https://doi.org/10.1051/0004-6361/201323014

187. Southworth, J., Evans, D.F.: Contamination from a nearby star cannot explain the anomalous transmission spectrum of the ultrashort period giant planet WASP-103 b. MNRAS 463(1), 37 (2016). https://doi.org/10.1093/mnras/stw1943

188. Sato, B., Hartman, J.D., Bakos, G.Á. ., Béky, B., Torres, G., Latham, D.W., Kovács, G., Csubry, Z., Penev, K., Noyes, R.W., Buchhave, L.A., Quinn, S.N., Everett, M., Esquerdo, G.A., Fischer, D.A., Howard, A.W., Johnson, J.A., Marcy, G.W., Sasselov, D.D., Szklenár, T., Lázár, J., Papp, I., Sári, P.: HAT-P-38b: A saturn-mass planet transiting a late G star. Publ. Astron. Soc. Jpn. 64, 97 (2012). https://doi.org/10.1093/pasj/64.5.97

189. Southworth, J., Mancini, L., Novati, S.C., Dominik, M., Glitrup, M., Hinse, T.C., Jørgensen, U.G., Mathiasen, M., Ricci, D., Maier, G., Zimmer, F., Bozza, V., Browne, P., Bruni, I., Burgdorf, M., Dall'Ora, M., Finet, F., Harpsøe, K., Hundertmark, M., Liebig, C., Rahvar, S., Scarpetta, G., Skottfelt, J., Smalley, B., Snodgrass, C., Surdej, J.: High-precision photometry by telescope defocusing - III. The transiting planetary system WASP-2. MNRAS 408(3), 1680 (2010). https://doi.org/10.1111/j.1365-2966.2010.17238.x

190. Anderson, D.R., Collier Cameron, A., Delrez, L., Doyle, A.P., Gillon, M., Hellier, C., Jehin, E., Lendl, M., Maxted, P.F.L., Madhusudhan, N., Pepe, F., Pollacco, D., Queloz, D., Ségransan, D., Smalley, B., Smith, A.M.S., Triaud, A.H.M.J., Turner, O.D., Udry, S., West, R.G.: The discoveries of WASP-91b, WASP-105b and WASP-107b: Two warm Jupiters and a planet in the transition region between ice giants and gas giants. Astron. Astrophys. 604, A110 (2017). https://doi.org/10.1051/0004-6361/201730439

191. Močnik, T., Hellier, C., Anderson, D.R., Clark, B.J.M., Southworth, J.: Starspots on WASP-107 and pulsations of WASP-118. MNRAS 469(2), 1622 (2017). https://doi.org/10.1093/mnras/stx972

192. Hartman, J.D., Bakos, G.Á.., Béky, B., Torres, G., Latham, D.W., Csubry, Z., Penev, K., Shporer, A., Fulton, B.J., Buchhave, L.A., Johnson, J.A., Howard, A.W., Marcy, G.W., Fischer, D.A., Kovács, G., Noyes, R.W., Esquerdo, G.A., Everett, M., Szklenár, T., Quinn, S.N., Bieryla, A., Knox, R.P., Hinz, P., Sasselov, D.D., Fűrész, G., Stefanik, R.P., Lázár, J., Papp, I., Sári, P.: HAT-P-39b-HAT-P-41b: Three highly inflated transiting hot Jupiters. AJ 144(5), 139 (2012). https://doi.org/10.1088/0004-6256/144/5/139

193. Christian, D.J., Gibson, N.P., Simpson, E.K., Street, R.A., Skillen, I., Pollacco, D., Collier Cameron, A., Joshi, Y.C., Keenan, F.P., Stempels, H.C., Haswell, C.A., Horne, K., Anderson, D.R., Bentley, S., Bouchy, F., Clarkson, W.I., Enoch, B., Hebb, L., Hébrard, G., Hellier, C., Irwin, J., Kane, S.R., Lister, 
T.A., Loeillet, B., Maxted, P., Mayor, M., McDonald, I., Moutou, C., Norton, A.J., Parley, N., Pont, F., Queloz, D., Ryans, R., Smalley, B., Smith, A.M.S., Todd, I., Udry, S., West, R.G., Wheatley, P.J., Wilson, D.M.: WASP-10b: a 3M $\mathrm{M}_{J}$, gas-giant planet transiting a late-type K star. MNRAS 392(4), 1585 (2009). https://doi.org/10.1111/j.1365-2966.2008.14164.x

194. Johnson, J.A., Winn, J.N., Cabrera, N.E., Carter, J.A.: A smaller radius for the transiting exoplanet WASP-10b. Astrophys. J. Lett. 692(2), L100 (2009). https://doi.org/10.1088/0004-637X/692/2/L100

195. Hartman, J.D., Bakos, G.Á.., Torres, G., Kovács, G., Johnson, J.A., Howard, A.W., Marcy, G.W., Latham, D.W., Bieryla, A., Buchhave, L.A., Bhatti, W., Béky, B., Csubry, Z., Penev, K., de ValBorro, M., Noyes, R.W., Fischer, D.A., Esquerdo, G.A., Everett, M., Szklenár, T., Zhou, G., Bayliss, D., Shporer, A., Fulton, B.J., Sanchis-Ojeda, R., Falco, E., Lázár, J., Papp, I., Sári, P.: HAT-P44b, HAT-P-45b, and HAT-P-46b: Three transiting hot Jupiters in possible multi-planet systems. AJ 147(6), 128 (2014). https://doi.org/10.1088/0004-6256/147/6/128

196. West, R.G., Collier Cameron, A., Hebb, L., Joshi, Y.C., Pollacco, D., Simpson, E., Skillen, I., Stempels, H.C., Wheatley, P.J., Wilson, D., Anderson, D., Bentley, S., Bouchy, F., Christian, D., Enoch, B., Gibson, N., Hébrard, G., Hellier, C., Loeillet, B., Mayor, M., Maxted, P., McDonald, I., Moutou, C., Pont, F., Queloz, D., Smith, A.M.S., Smalley, B., Street, R.A., Udry, S.: The sub-Jupiter mass transiting exoplanet WASP-11b. Astron. Astrophys. 502(1), 395 (2009). https://doi.org/10.1051/0004-6361/200810973

197. Wang, X.B., Gu, S.H., Collier Cameron, A., Wang, Y.b., Hui, H.K., Kwok, C.T., Yeung, B., Leung, K.C.: The refined physical properties of transiting exoplanetary system WASP-11/HAT-P-10. AJ 147(4), 92 (2014). https://doi.org/10.1088/0004-6256/147/4/92

198. Lam, K.W.F., Faedi, F., Brown, D.J.A., Anderson, D.R., Delrez, L., Gillon, M., Hébrard, G., Lendl, M., Mancini, L., Southworth, J., Smalley, B., Triaud, A.H.M., Turner, O.D., Hay, K.L., Armstrong, D.J., Barros, S.C.C., Bonomo, A.S., Bouchy, F., Boumis, P., Collier Cameron, A., Doyle, A.P., Hellier, C., Henning, T., Jehin, E., King, G., Kirk, J., Louden, T., Maxted, P.F.L., McCormac, J.J., Osborn, H.P., Palle, E., Pepe, F., Pollacco, D., Prieto-Arranz, J., Queloz, D., Rey, J., Ségransan, D., Udry, S., Walker, S., West, R.G., Wheatley, P.J.: From dense hot Jupiter to low-density Neptune: The discovery of WASP-127b, WASP-136b, and WASP-138b. Astron. Astrophys. 599, A3 (2017). https://doi.org/10.1051/0004-6361/201629403

199. Palle, E., Chen, G., Prieto-Arranz, J., Nowak, G., Murgas, F., Nortmann, L., Pollacco, D., Lam, K., Montanes-Rodriguez, P., Parviainen, H., Casasayas-Barris, N.: Feature-rich transmission spectrum for WASP-127b. Cloud-free skies for the puffiest known super-Neptune? Astron. Astrophys. 602, L15 (2017). https://doi.org/10.1051/0004-6361/201731018

200. Hebb, L., Collier-Cameron, A., Loeillet, B., Pollacco, D., Hébrard, G., Street, R.A., Bouchy, F., Stempels, H.C., Moutou, C., Simpson, E., Udry, S., Joshi, Y.C., West, R.G., Skillen, I., Wilson, D.M., McDonald, I., Gibson, N.P., Aigrain, S., Anderson, D.R., Benn, C.R., Christian, D.J., Enoch, B., Haswell, C.A., Hellier, C., Horne, K., Irwin, J., Lister, T.A., Maxted, P., Mayor, M., Norton, A.J., Parley, N., Pont, F., Queloz, D., Smalley, B., Wheatley, P.J.: WASP-12b: The hottest transiting extrasolar planet yet discovered. ApJ 693(2), 1920 (2009). https://doi.org/10.1088/0004-637X/693/2/1920

201. Bieryla, A., Hartman, J.D., Bakos, G.Á. ., Bhatti, W., Kovács, G., Boisse, I., Latham, D.W., Buchhave, L.A., Csubry, Z., Penev, K., de Val-Borro, M., Béky, B., Falco, E., Torres, G., Noyes, R.W., Berlind, P., Calkins, M.C., Esquerdo, G.A., Lázár, J., Papp, I., Sári, P.: HAT-P-49b: A 1.7 M $J$ planet transiting a bright 1.5 M ${ }_{s}$ F-star. AJ 147(4), 84 (2014). https://doi.org/10.1088/0004-6256/147/4/84

202. Temple, L.Y., Hellier, C., Albrow, M.D., Anderson, D.R., Bayliss, D., Beatty, T.G., Bieryla, A., Brown, D.J.A., Cargile, P.A., Collier Cameron, A., Collins, K.A., Colón, K.D., Curtis, I.A., D’ Ago, G., Delrez, L., Eastman, J., Gaudi, B.S., Gillon, M., Gregorio, J., James, D., Jehin, E., Joner, M.D., Kielkopf, J.F., Kuhn, R.B., Labadie-Bartz, J., Latham, D.W., Lendl, M., Lund, M.B., Malpas, A.L., Maxted, P.F.L., Myers, G., Oberst, T.E., Pepe, F., Pepper, J., Pollacco, D., Queloz, D., Rodriguez, J.E., Ségransan, D., Siverd, RJ., Smalley, B., Stassun, K.G., Stevens, D.J., Stockdale, C., Tan, T.G., Triaud, A.H.M.J., Udry, S., Villanueva, S., West, R.G., Zhou, G.: WASP-167b/KELT-13b: joint discovery of a hot Jupiter transiting a rapidly rotating F1V star. MNRAS 471(3), 2743 (2017). https://doi.org/10.1093/mnras/stx1729

203. West, R.G., Anderson, D.R., Gillon, M., Hebb, L., Hellier, C., Maxted, P.F.L., Queloz, D., Smalley, B., Triaud, A.H.M.J., Wilson, D.M., Bentley, S.J., Collier Cameron, A., Enoch, B., Horne, K., Irwin, J., Lister, T.A., Mayor, M., Parley, N., Pepe, F., Pollacco, D., Segransan, D., Spano, M., Udry, S., Wheatley, P.J.: The low density transiting exoplanet WASP-15b. AJ 137(6), 4834 (2009). https://doi.org/10.1088/0004-6256/137/6/4834 
204. Hebb, L., Collier-Cameron, A., Triaud, A.H.M.J., Lister, T.A., Smalley, B., Maxted, P.F.L., Hellier, C., Anderson, D.R., Pollacco, D., Gillon, M., Queloz, D., West, R.G., Bentley, S., Enoch, B., Haswell, C.A., Horne, K., Mayor, M., Pepe, F., Segransan, D., Skillen, I., Udry, S., Wheatley, P.J.: WASP-19b: The shortest period transiting exoplanet yet discovered. ApJ 708(1), 224 (2010). https://doi.org/10.1088/0004-637X/708/1/224

205. Wong, I., Knutson, H.A., Cowan, N.B., Lewis, N.K., Agol, E., Burrows, A., Deming, D., Fortney, J.J., Fulton, B.J., Langton, J., Laughlin, G., Showman, A.P.: Constraints on the atmospheric circulation and variability of the eccentric hot Jupiter XO-3b. ApJ 794(2), 134 (2014). https://doi.org/10.1088/0004-637X/794/2/134

206. Bouchy, F., Hebb, L., Skillen, I., Collier Cameron, A., Smalley, B., Udry, S., Anderson, D.R., Boisse, I., Enoch, B., Haswell, C.A., Hébrard, G., Hellier, C., Joshi, Y., Kane, S.R., Maxted, P.F.L., Mayor, M., Moutou, C., Pepe, F., Pollacco, D., Queloz, D., Ségransan, D., Simpson, E.K., Smith, A.M.S., Stempels, H.C., Street, R., Triaud, A.H.M.J., West, R.G., Wheatley, P.J.: WASP21b: a hot-Saturn exoplanet transiting a thick disc star. Astron. Astrophys. 519, A98 (2010). https://doi.org/10.1051/0004-6361/201014817

207. Street, R.A., Simpson, E., Barros, S.C.C., Pollacco, D., Joshi, Y., Todd, I., Collier Cameron, A., Enoch, B., Parley, N., Stempels, E., Hebb, L., Triaud, A.H.M.J., Queloz, D., Segransan, D., Pepe, F., Udry, S., Lister, T.A., Depagne, É., West, R.G., Norton, A.J., Smalley, B., Hellier, C., Anderson, D.R., Maxted, P.F.L., Bentley, S.J., Skillen, I., Gillon, M., Wheatley, P., Bento, J., CathawayKjontvedt, P., Christian, D.J.: WASP-24 b: A new transiting close-in hot Jupiter orbiting a late F-star. ApJ 720(1), 337 (2010). https://doi.org/10.1088/0004-637X/720/1/337

208. Burke, C.J., McCullough, P.R., Valenti, J.A., Long, D., Johns-Krull, C.M., Machalek, P., Janes, K.A., Taylor, B., Fleenor, M.L., Foote, C.N., Gary, B.L., García-Melendo, E., Gregorio, J., Vanmunster, T.: XO-5b: A Transiting Jupiter-sized Planet with a 4 Day Period. ApJ 686(2), 1331 (2008). https://doi.org/10.1086/591497

209. Hartman, J.D., Bakos, G.Á.., Buchhave, L.A., Torres, G., Latham, D.W., Kovács, G., Bhatti, W., Csubry, Z., de Val-Borro, M., Penev, K., Huang, C.X., Béky, B., Bieryla, A., Quinn, S.N., Howard, A.W., Marcy, G.W., Johnson, J.A., Isaacson, H., Fischer, D.A., Noyes, R.W., Falco, E., Esquerdo, G.A., Knox, R.P., Hinz, P., Lázár, J., Papp, I., Sári, P.: HAT-P-57b: A short-period giant planet transiting a bright rapidly rotating a8v star confirmed via doppler tomography. AJ 150(6), 197 (2015). https://doi.org/10.1088/0004-6256/150/6/197

210. Enoch, B., Cameron, A.C., Anderson, D.R., Lister, T.A., Hellier, C., Maxted, P.F.L., Queloz, D., Smalley, B., Triaud, A.H.M.J., West, R.G., Brown, D.J.A., Gillon, M., Hebb, L., Lendl, M., Parley, N., Pepe, F., Pollacco, D., Segransan, D., Simpson, E., Street, R.A., Udry, S.: WASP-25b: a $0.6 \mathrm{M}_{J}$ planet in the Southern hemisphere. MNRAS 410(3), 1631 (2011). https://doi.org/10.1111/j.1365-2966.2010.17550.x

Publisher's note Springer Nature remains neutral with regard to jurisdictional claims in published maps and institutional affiliations. 


\section{Affiliations}

Anastasia Kokori ${ }^{1,2} \cdot$ Angelos Tsiaras $^{1,2} \cdot$ Billy Edwards $^{1} \cdot$ Marco Rocchetto ${ }^{1,3}$. Giovanna Tinetti ${ }^{1}$. Anaël Wünsche ${ }^{4}$. Nikolaos Paschalis ${ }^{5}$. Vikrant Kumar Agnihotri ${ }^{6}$. Matthieu Bachschmidt ${ }^{7}$. Marc Bretton ${ }^{4}$. Hamish Caines ${ }^{1}$. Mauro Caló ${ }^{32}$. Roland Casali ${ }^{8}$. Martin Crow ${ }^{9,10}$. Simon Dawes ${ }^{9,10}$. Marc Deldem ${ }^{11}$. Dimitrios Deligeorgopoulos ${ }^{12}$. Roger Dymock ${ }^{9}$. Phil Evans ${ }^{13}$. Carmelo Falco ${ }^{14}$. Stephane Ferratfiat ${ }^{4}$. Martin Fowler ${ }^{9}$. Stephen Futcher ${ }^{15}$. Pere Guerra ${ }^{16}$. Francois Hurter ${ }^{17}$. Adrian Jones ${ }^{9} \cdot$ Wonseok Kang ${ }^{18} \cdot$ Taewoo Kim $^{18} \cdot$ Richard Lee $^{7}$. Claudio Lopresti ${ }^{19}$. Antonio Marino ${ }^{20}$. Matthias Mallonn ${ }^{21}$. Fabio Mortari ${ }^{22}$. Mario Morvan ${ }^{1}$. Lorenzo V. Mugnai ${ }^{33}$. Alessandro Nastasi ${ }^{14}$.

Valère Perroud ${ }^{23}$. Cédric Pereira ${ }^{34}$. Mark Phillips ${ }^{9,24}$. Pavel Pintr ${ }^{25}$. Manfred Raetz ${ }^{26}$. Francois Regembal ${ }^{27}$. John Savage ${ }^{9}$. Danilo Sedita ${ }^{28}$. Nick Sioulas ${ }^{29}$. lakovos Strikis ${ }^{30}$. Geoffrey Thurston ${ }^{9}$. Andrea Tomacelli ${ }^{20}$. Alberto Tomatis ${ }^{31}$

1 University College London, Gower Street, London, WC1E 6BT, UK

2 Aristotle University of Thessaloniki, University Campus, 54124, Thessaloniki, Greece

3 Telescope Live, Spaceflux Ltd, 71-75 Shelton Street, London, WC2H 9JQ, UK

4 Observatoire des Baronnies Provençales, Le Mas des Grés, Route de Nyons, 05150 Moydans, France

5 Nunki Observatory (private observatory), Skiathos, Greece

6 Cepheid Observatory (private observatory), Rawatbhata, India

7 Amateur Astronomer, London, United Kingdom

8 Alto2000 Observatory, Rome, Italy

9 British Astronomical Association, Burlington House, Piccadilly, Mayfair, London, W1J 0DU, UK

10 Crayford Manor House Astronomical Society Dartford, Parsonage Lane Pavilion, Parsonage Lane, Sutton-at-Hone, Dartford, Kent, DA4 9HD, UK

11 Les Barres Observatory (private observatory), Lamanon, France

12 Artemis Observatory (private observatory), Evrytania, Greece

13 El Sauce Observatory (private observatory), Coquimbo, Chile

14 GAL Hassin - Centro Internazionale per le Scienze Astronomiche, Via della Fontana Mitri, 90010, Isnello, Palermo, Italy

15 Hampshire Astronomical Group, Hinton Manor Ln, Clanfield, Waterlooville PO8 0QR, UK

16 Observatori Astronòmic Albanyà (private observatory), Girona, Spain

17 Albireo Observatory (private observatory), Lajoux, Switzerland

18 National Youth Space Center, 11-1 Deokheung-ri, Dongil-myeon, Goheung-gun, Jeollanam-do, South Korea

19 GAD - Gruppo Astronomia Digitale, La Spezia, Italy

20 Unione Astrofili Napoletani, Salita Moiariello, 16, CAP 80131 Napoli NA, Italy

21 Leibniz Institute for Astrophysics Potsdam (AIP), An der Sternwarte 16, 14482 Potsdam, Germany

22 Hypatia Observatory (private observatory), Rimini, Italy

23 Observatoire de Duines (private observatory), Savoie, France

24 Astronomical Society of Edinburgh, Edinburgh, UK 
25 Institute of Plasma Physics AS CR, v. v. i., TOPTEC centre, Sobotecka 1660, 51101 Turnov, Czechia

26 Herges-Hallenberg Observatory (private observatory), Herges-Hallenberg, Germany

27 HRT Observatory (private observatory), Badajoz, Spain

28 Osservatorio Sedita Castrofilippo (private observatory), Castrofilippo, Italy

29 NOAK Observatory (private observatory), Ioannina, Greece

30 Hellenic Amateur Astronomy Association, Athens, Greece

31 Alto-Observatory (private observatory), Rome, Italy

32 Cavallino Observatory (private observatory), Tuscany, Italy

33 La Sapienza Universita di Roma, Department of Physics, Piazzale Aldo Moro 2, 00185 Roma, Italy

34 Instituto de Astrofísica e Ciências do Espaço, Departamento de Física, Faculdade de Ciências, Universidade de Lisboa, Campo Grande, PT1749-016 Lisboa, Portugal 